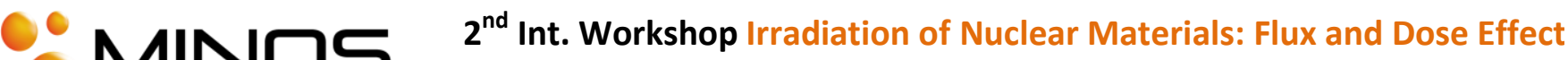 MIN口S

\section{Evolution under Irradiation of Optimized Austenitic Steel For Gen-IV Reactors. Impact on Fuel Cladding Properties and Performances.}

\author{
Arnaud COURCELLE ${ }^{1}$ Caroline BISOR ${ }^{1}$, Emma PIOZIN ${ }^{1}$, Mikael KOUNTCHOU ${ }^{1}$, \\ Pierre GAVOILLE ${ }^{1}$, Marion LE FLEM ${ }^{2}$, Jean-Louis SÉRAN ${ }^{2}$ \\ ${ }^{1}$ CEA-DEN-DMN, Service d'Etudes des Matériaux Irradiés, SEMI (Saclay, France) \\ ${ }^{2}$ CEA-DEN-DMN, Department of Nuclear Materials (Saclay, France)
}

An important class of swelling-resistant alloys used in fast-reactor are the cold-worked, titaniumstabilized austenitic stainless steels (15-15Ti AIM1 in France, DIN1.4970 in Germany, optimized D9 in the US and AFIC1 in India). An advanced 15-15Ti alloy was selected for the fuel cladding of the initial core of the fast-reactor French project ASTRID [1]. To extend its qualification at high dose, post-irradiation examinations were recently performed on mixed-oxide fuel pins, irradiated in the Phénix fast-reactor up to $130 \mathrm{dpa}$ and retrieved after Phénix shutdown. As shown in Fig. 1, destructive examinations in hot labs (CEA/LECA-STAR and CEA/LECI facilities) were done to quantify the contribution of irradiation creep and swelling to the total cladding deformation. The results are compared with the past examinations on the same material at lower dose 80 and $110 \mathrm{dpa}$ [2] and confirm the very good swelling resistance of this optimized alloy at $130 \mathrm{dpa}$.
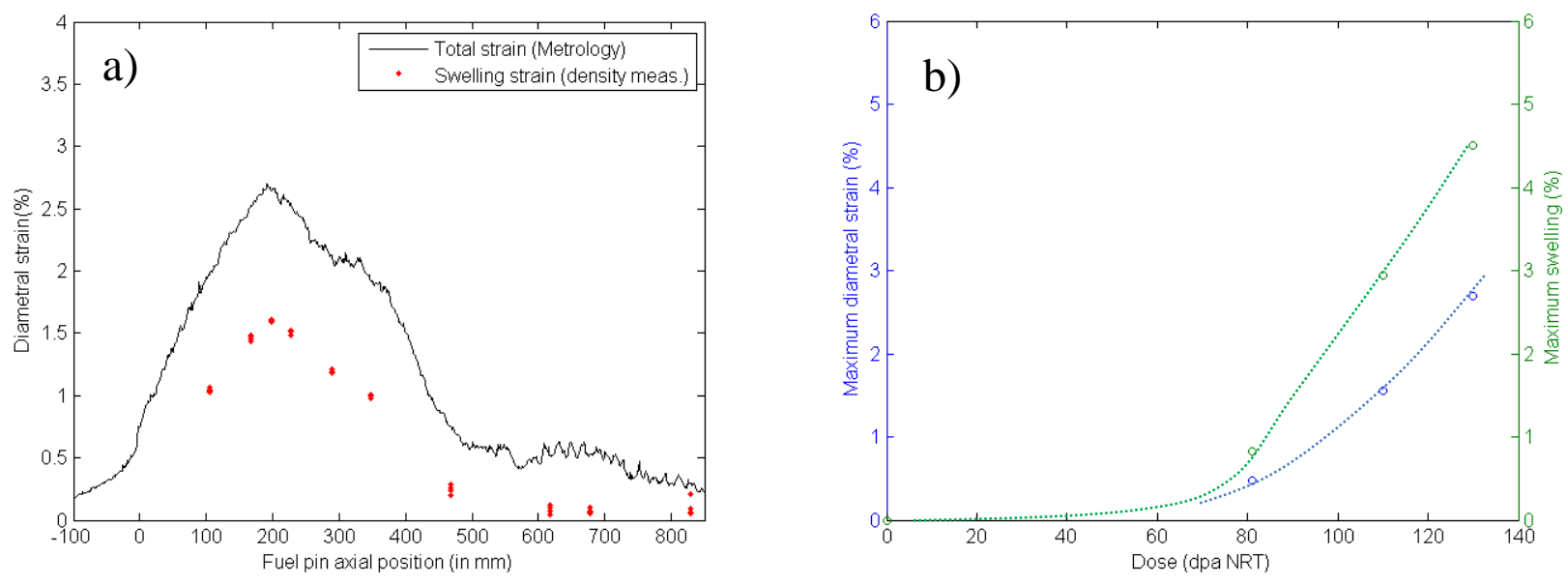

Fig. 1: a) Diametral strain of an irradiated 15-15Ti (optimized) cladding at a maximum dose of 130 dpa. b) Maximum diametral strain and swelling versus the maximum cladding dose.

The evolution of $15-15 \mathrm{Ti}$ microstructure under neutron irradiation was investigated by TEM [3]. A shown in Fig. 2, the irradiated microstructure features complex interactions between vacancy clusters, precipitates (fine titanium-carbide and coarser $\mathrm{Cr}$-rich precipitates, $\mathrm{G}$ phase) and dislocations network. The simulation of irradiation effects on advanced 15-15Ti using ions irradiation in the JANNUS-Saclay facility will be presented. The comparison between single $\left(2 \mathrm{MeV} \mathrm{Fe}^{2+}\right)$ and dual beams ( $2 \mathrm{MeV} \mathrm{Fe}^{2+}-1 \mathrm{MeV} \mathrm{He}^{+}$) experiments stresses the importance of helium in swelling mechanisms. As in neutron irradiation, heterogeneous nucleation of vacancy clusters is evidenced but marked differences in precipitation mechanisms are observed between neutron and ions irradiation. 

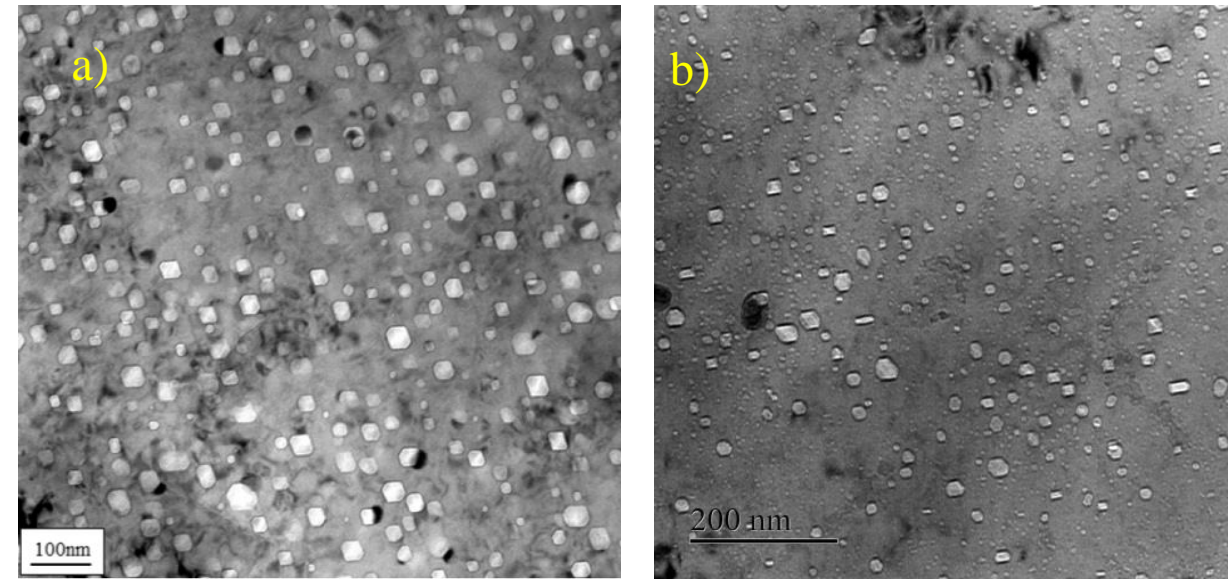

Fig. 2: TEM micrographs of optimized 15-15Ti samples showing cavities microstructure and the interaction between precipitates and voids : a) neutron irradiation $88 \mathrm{dpaNRT} 470^{\circ} \mathrm{C}, \mathrm{b}$ ) ions irradiation $90 \mathrm{dpaKP} 630^{\circ} \mathrm{C}$.

The modeling of irradiation evolution in such complex alloy is challenging. The presentation will summarize the current efforts to develop a model capable of predicting temperature, dose, and flux effects on swelling using cluster-dynamics techniques. The modeling of helium effects is also discussed. One of the future areas of work is to include the effects of precipitation (interface effects, helium and point-defects trapping, solid-solution decay etc.).

The Impact of irradiation on tensile and creep properties of 15-15Ti is also briefly discussed with special emphasis on correlations between swelling and embrittlement. At high temperature $>600^{\circ} \mathrm{C}$, limited data on cold-worked $316 \mathrm{Ti}$ and $15-15 \mathrm{Ti}$ shows that creep rupture time and ductility are reduced and intergranular failure is enhanced.

\section{References}

[1] Le Flem et al. "Status of the French R\&D on materials for the core components of ASTRID", International Congress on Advances in Nuclear Power Plants, ICAPP 2014, Charlotte, USA, April 6-9 2014.

[2] A. Maillard et al. "Swelling and Irradiation Creep of Neutron Irradiated 316Ti and 15-15Ti steels", Effects of Radiation in Metals : $16^{\text {th }}$ International Symposium, ASTM STP 1175, 1992.

[3] C. Bisor et al., Personal communication CEA, 2012.

[4] M. Kountchou et al. "Ions Irradiations of Cladding Materials for the Fourth-Generation Nuclear Reactors", Material Science and Technoloy, MS\&T 2015, Colombus, USA, October 4-8 2015. 
DE LA RECHERCHE À L'INDUSTRIE

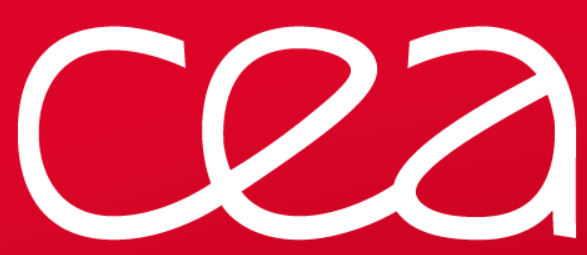

\section{EVOLUTION UNDER IRRADIATION OF OPTIMIZED AUSTENITIC STEEL FOR GENIV REACTOR.}

Minos, Cadarache November 4-6

Arnaud Courcelle

CEA-Saclay

DEN/DANS/DMN/SEMI/LCMI 


\section{Cea OUTLINE}

\section{MINDS}

- Neutron irradiation and Post-irradiation examinations

○ Ion irradiation

○ Modeling

- Impact of irradiation on mechanical properties 
MATERIALS 15-15TI 


\section{MATERIALS}

\section{MIN口S}

o Standard : CW 15-15Ti : used in standard French SFR Phenix assembly

\begin{tabular}{|l|l|l|l|l|l|l|l|l|}
\hline Lot & $\mathbf{C}$ & $\mathbf{M n}$ & $\mathbf{S i}$ & $\mathbf{C r}$ & $\mathbf{N i}$ & $\mathbf{M o}$ & $\mathbf{P}$ & $\mathbf{T i}$ \\
\hline Standard 15-15Ti & 0.08 & 1.0 & 0.3 & 14 & 14 & 1.3 & $<0.01$ & 0.3 \\
& 0.10 & 2.0 & 0.5 & 16 & 16 & 1.7 & 0.5 \\
\hline $\mathbf{1 5 - 1 5 T i}+\mathbf{S i}$ & 0.08 & 1.0 & 0.7 & 14 & 14 & 1.3 & $<0.01$ & 0.3 \\
& 0.10 & 2.0 & 0.9 & 16 & 16 & 1.7 & 0.5 \\
\hline $\mathbf{1 5 - 1 5 T i + S i + P}$ & 0.08 & 1.0 & 0.7 & 14 & 14 & 1.3 & 0.03 & 0.3 \\
& 0.10 & 2.0 & 0.9 & 16 & 16 & 1.7 & 0.05 & 0.5 \\
\hline
\end{tabular}

o CW 15-15Ti + Si : tested in SFR up to 130 dpa

o CW 15-15Ti + Si + P : AIM1, irradiated in Phénix up to 90 dpa post-irradiation examinations in progress 


\section{Cea UNIRRADIATED MATERIAL}

\section{MIN口S}

Fully austenitic, grain size $\sim 30 \mathrm{~mm}$ Thermal and mechanical twins, strongly strained grain TiN and TiMoC primary carbides No obvious texture

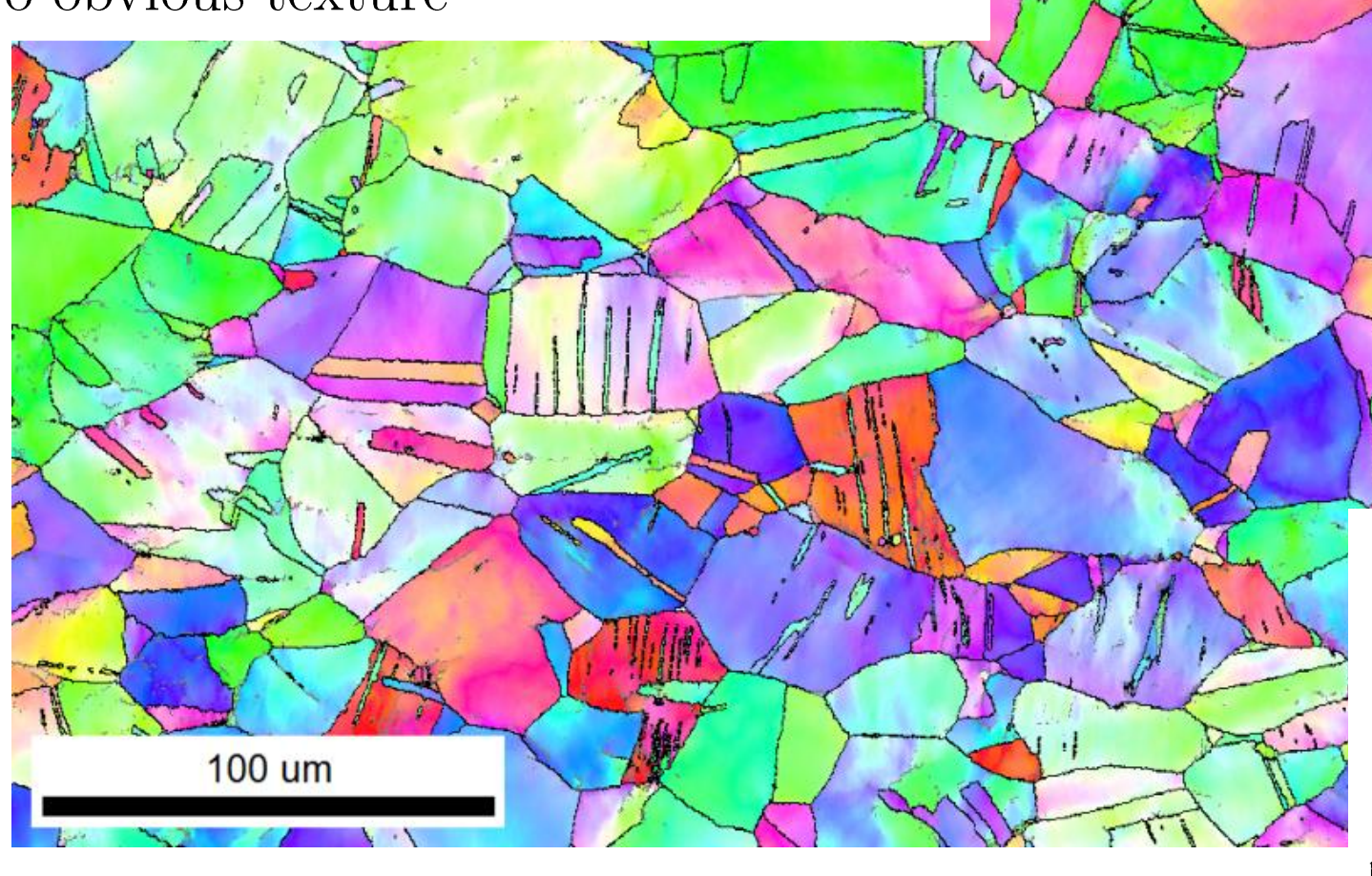

L. Mateus-Freire

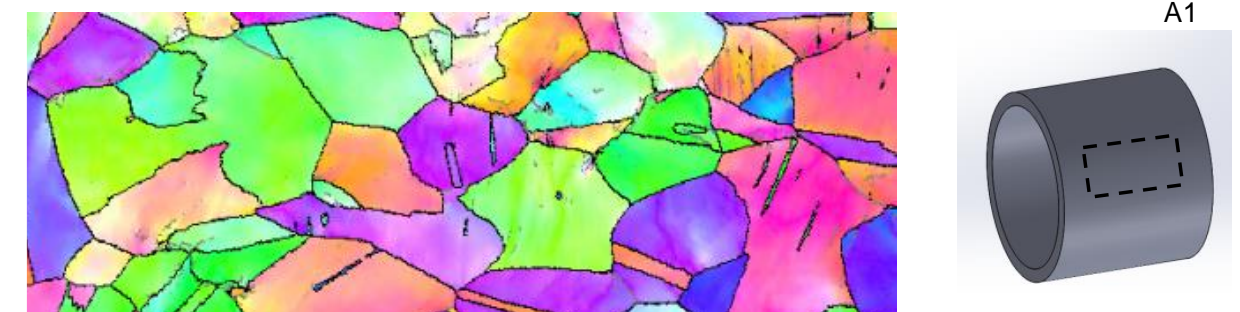

Austenite
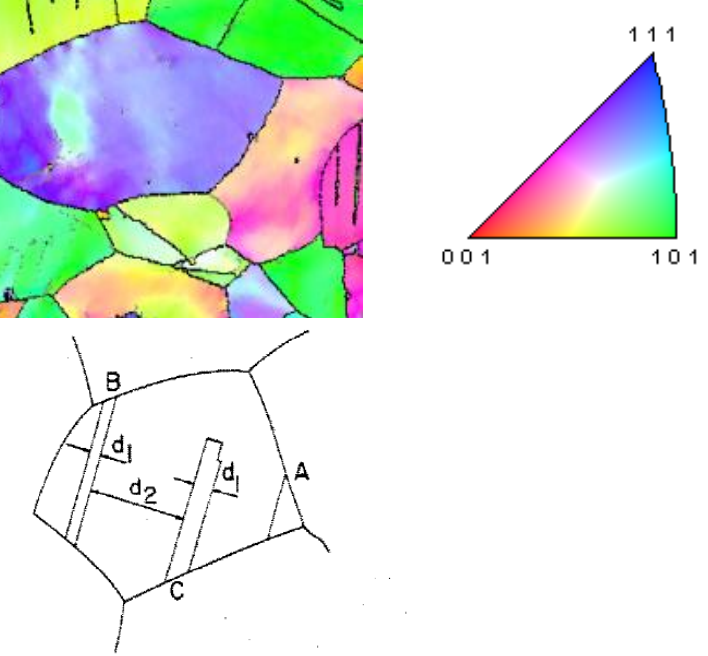


\section{Cea UNIRRADIATED MATERIAL}

\section{¿MINDS}

$$
\text { CW 15-15Ti }(+\mathrm{Si}+\mathrm{P})
$$
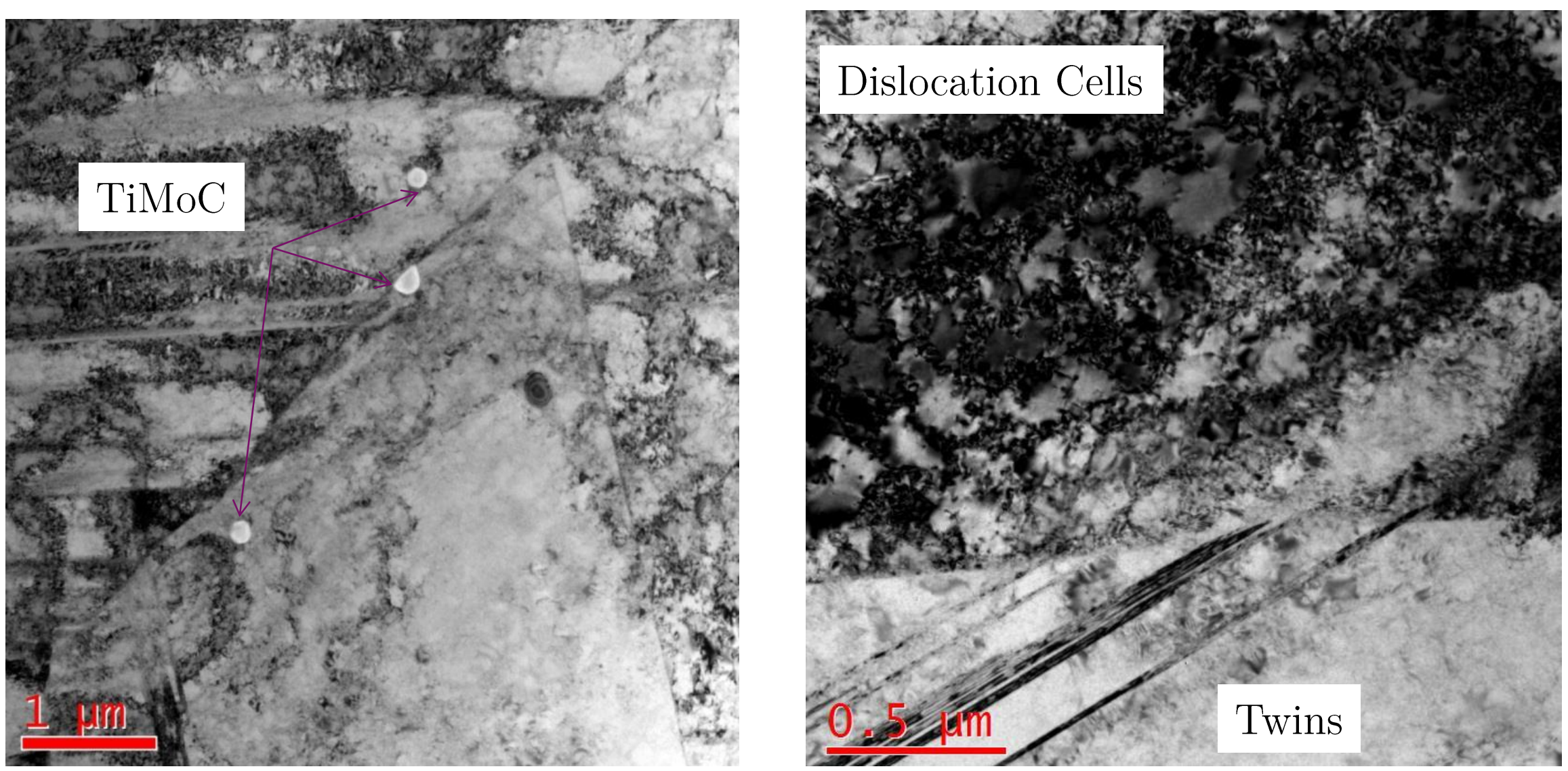


\section{Cea UNIRRADIATED + AGEING $650^{\circ} \mathrm{C}-24 \mathrm{H}$}

\section{$\because$ MIN口S}

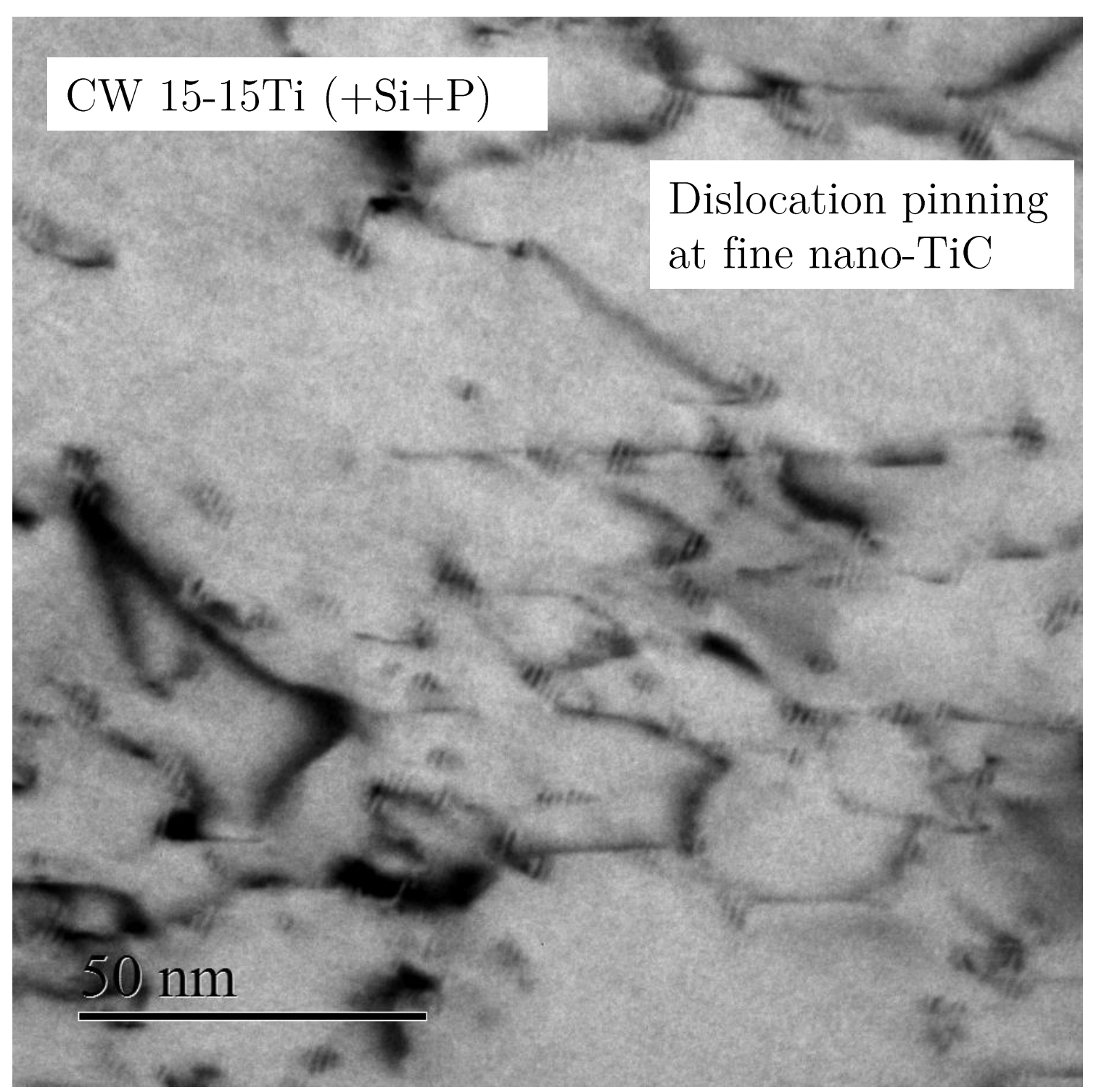




\section{Cea UNIRRADIATED + AGEING $850^{\circ} \mathrm{C}-100 \mathrm{H}$}

\section{¿MINDS}

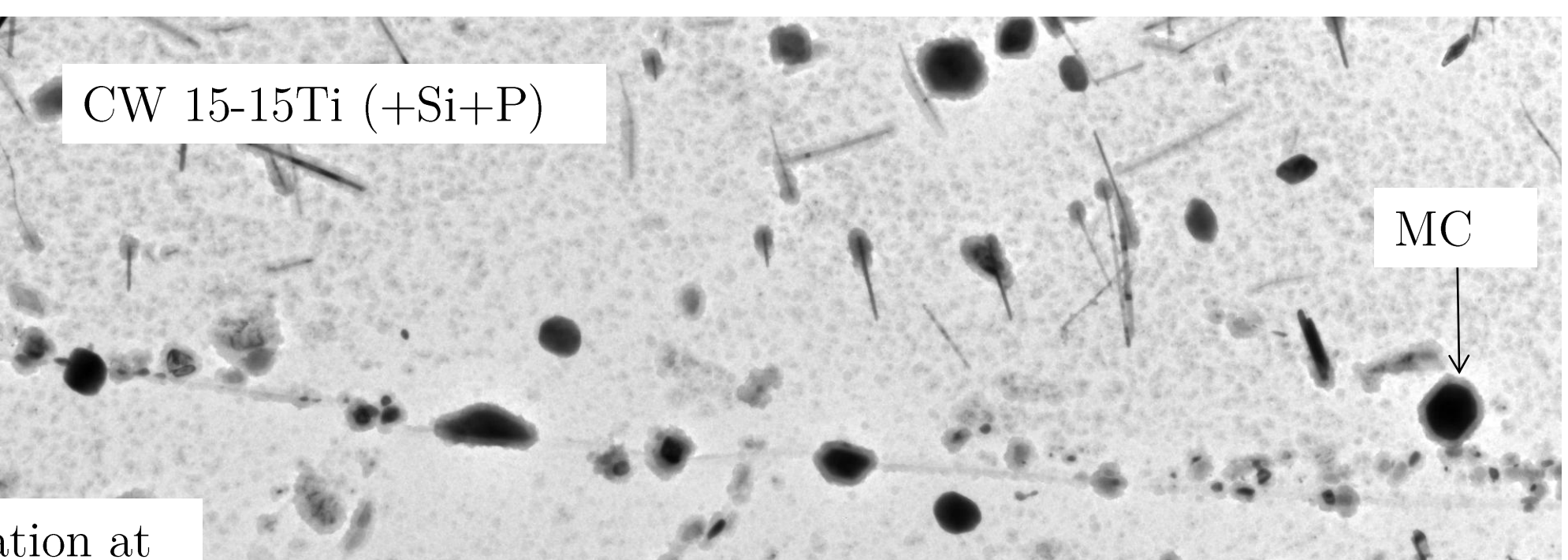

Precipitation at ex-twin during recrystallisation
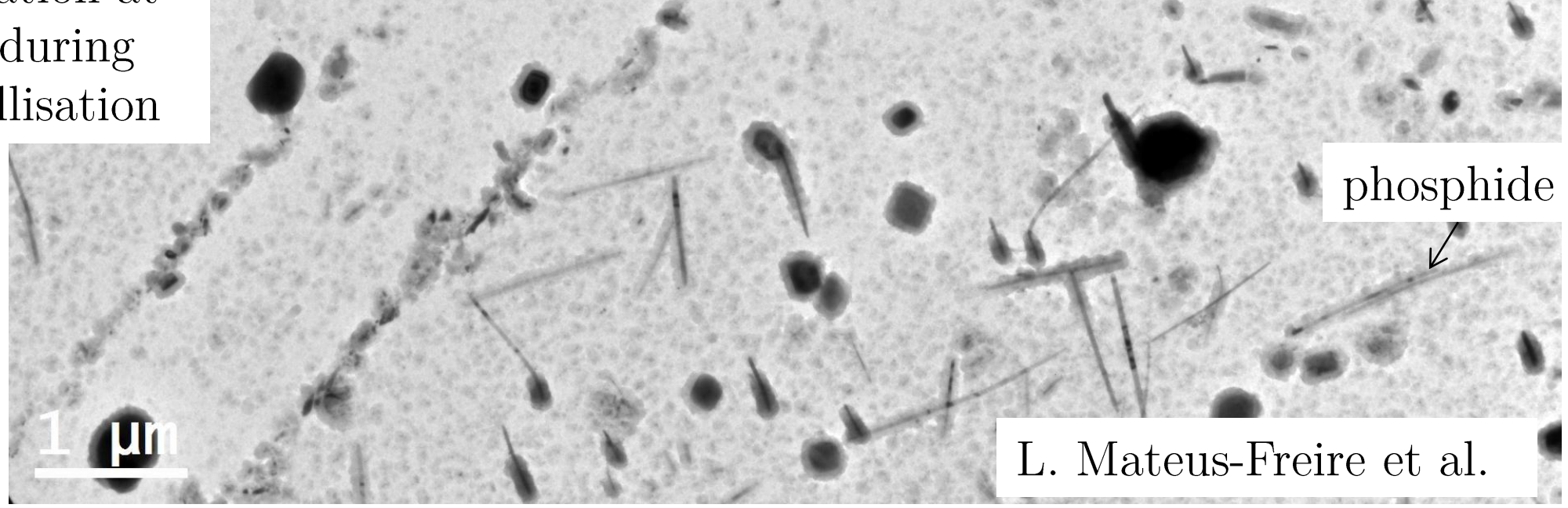

* phosphide


- $1, j$ 


\section{NEUTRON IRRADIATION}




\section{Cea NEUTRON IRRADIATION IN PHENIX}

\section{MIN口S}

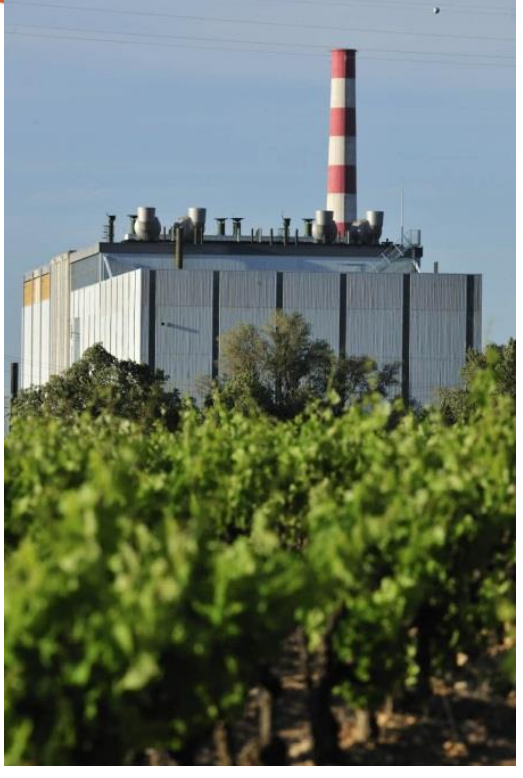

\section{After irradiation}



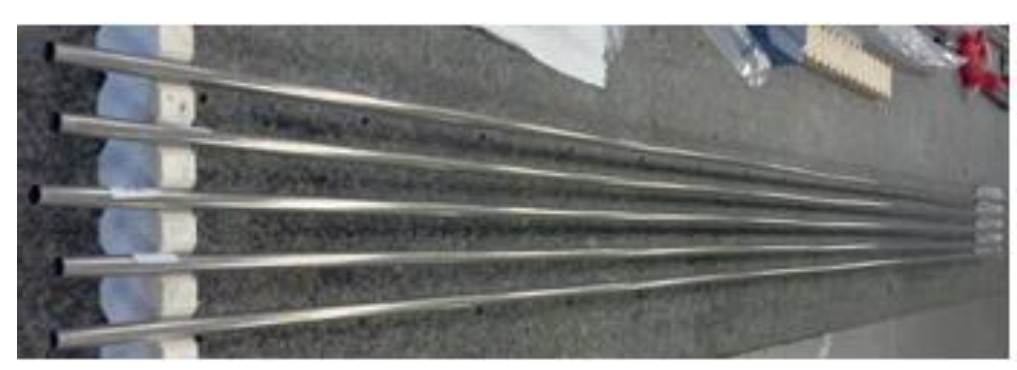

○ A long process!

- Cladding tube 15-15Ti+Si fabricated in 1981

- First irradiation from 1982 to 1985 up to 80 dpa

- Re-irradiation from 1986 to 1987 up to 110 dpa

- Third Re-irradiation up to 130 dpa from 1994 to 1998

- Assembly discharged in 2001

- PIE started in 2012 


\section{Cea NON DESTRUCTIVE AND DESTRUCTIVE EXAMINATION}

\section{$\because \mathrm{MINDS}$}

- CEA-Cadarache (LECA hot-lab facility)

- Metrology, $\gamma$ spectrometry

○ Metallography
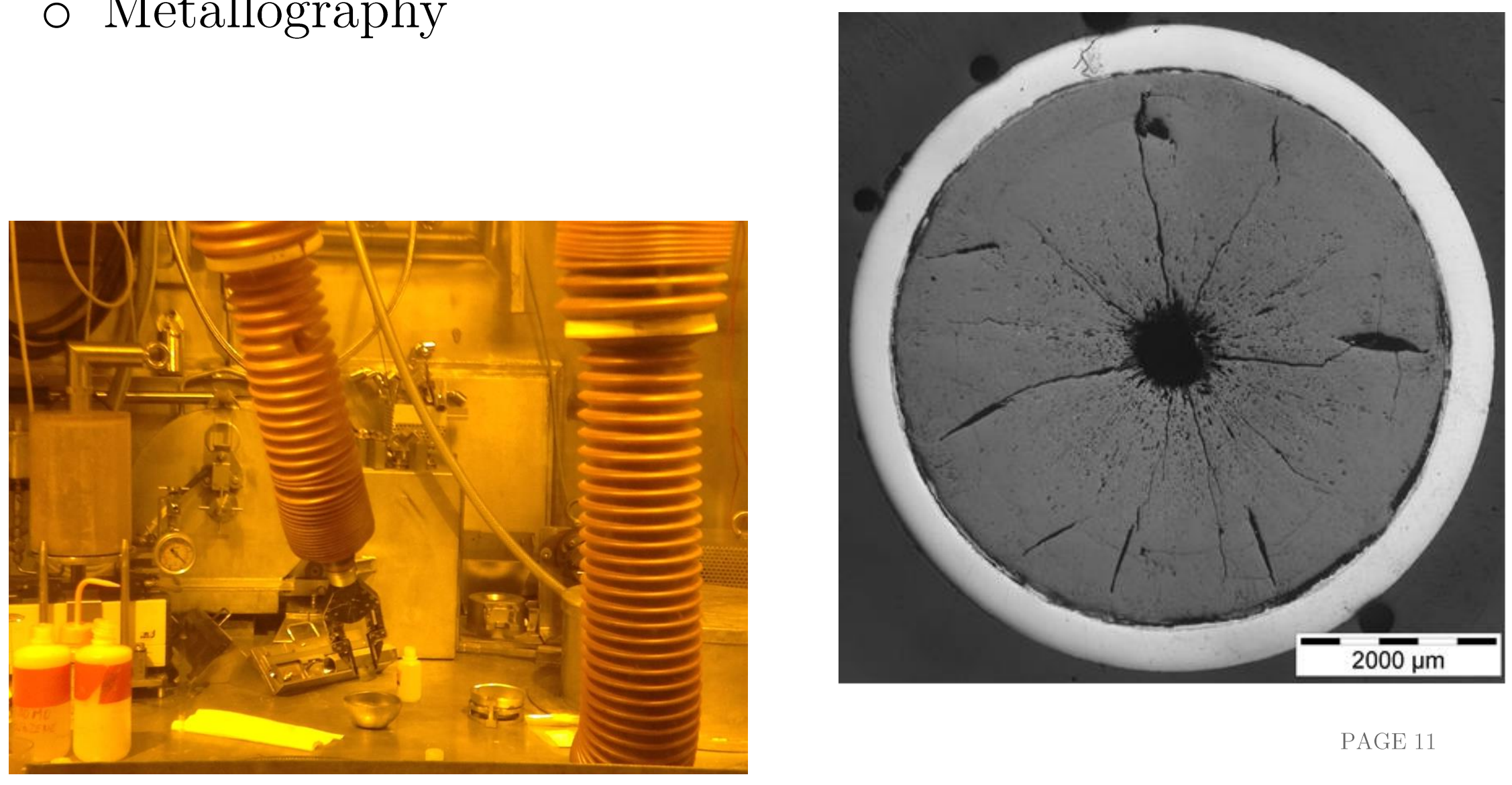


\section{CeA SAMPLE PREPARATION FOR DESTRUCTIVE EXAMINATION}

\section{$\because \mathrm{MINDS}$}

o Cutting (CEA-Cadarache - DEC - LECA - O. Descombin et al.)

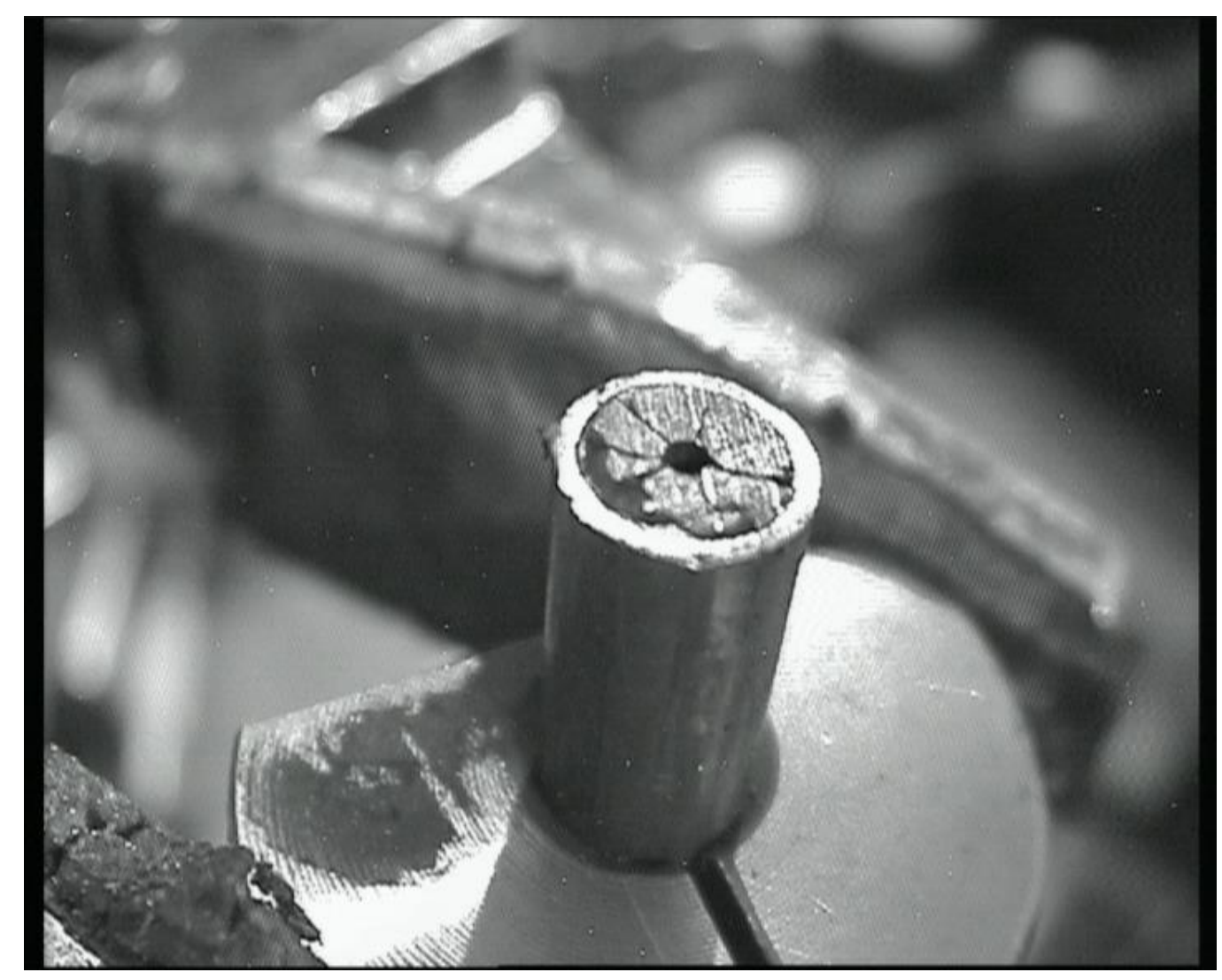




\section{cea SAMPLE PREPARATION FOR DESTRUCTIVE EXAMINATION}

\section{MINDS}

- Chemical dissolution HNO3 to remove fuel (CEA-Marcoule - E. Buravand et al.)






\section{SAMPLE PREPARATION FOR DESTRUCTIVE EXAMINATION}

\section{MINDS}

- Density measurements by immersion at CEA-Saclay-LECI (M. Sonnaert, P. Bottin et al.)

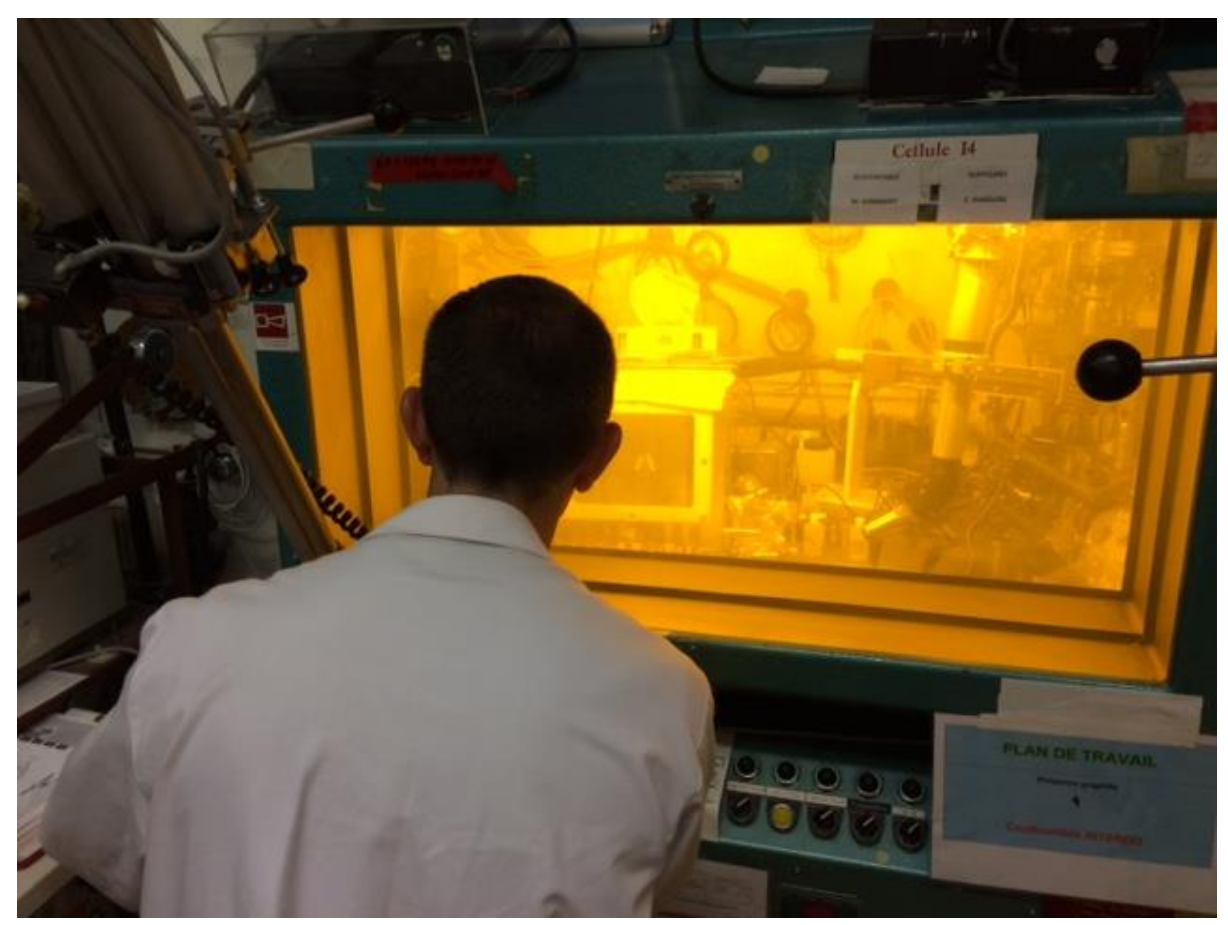




\section{NON-DESTRUCTIVE AND DESTRUCTIVE}

\section{MINDS}

○ Non destructive : diameter profile $\rightarrow$ total strain

o Destructive : Density by immersion $\rightarrow$ swelling



$\longleftrightarrow$ Fuel Column $\longrightarrow$ 


\section{Cea COMPARISON WITH PREVIOUS DATA}

\section{MINDS}

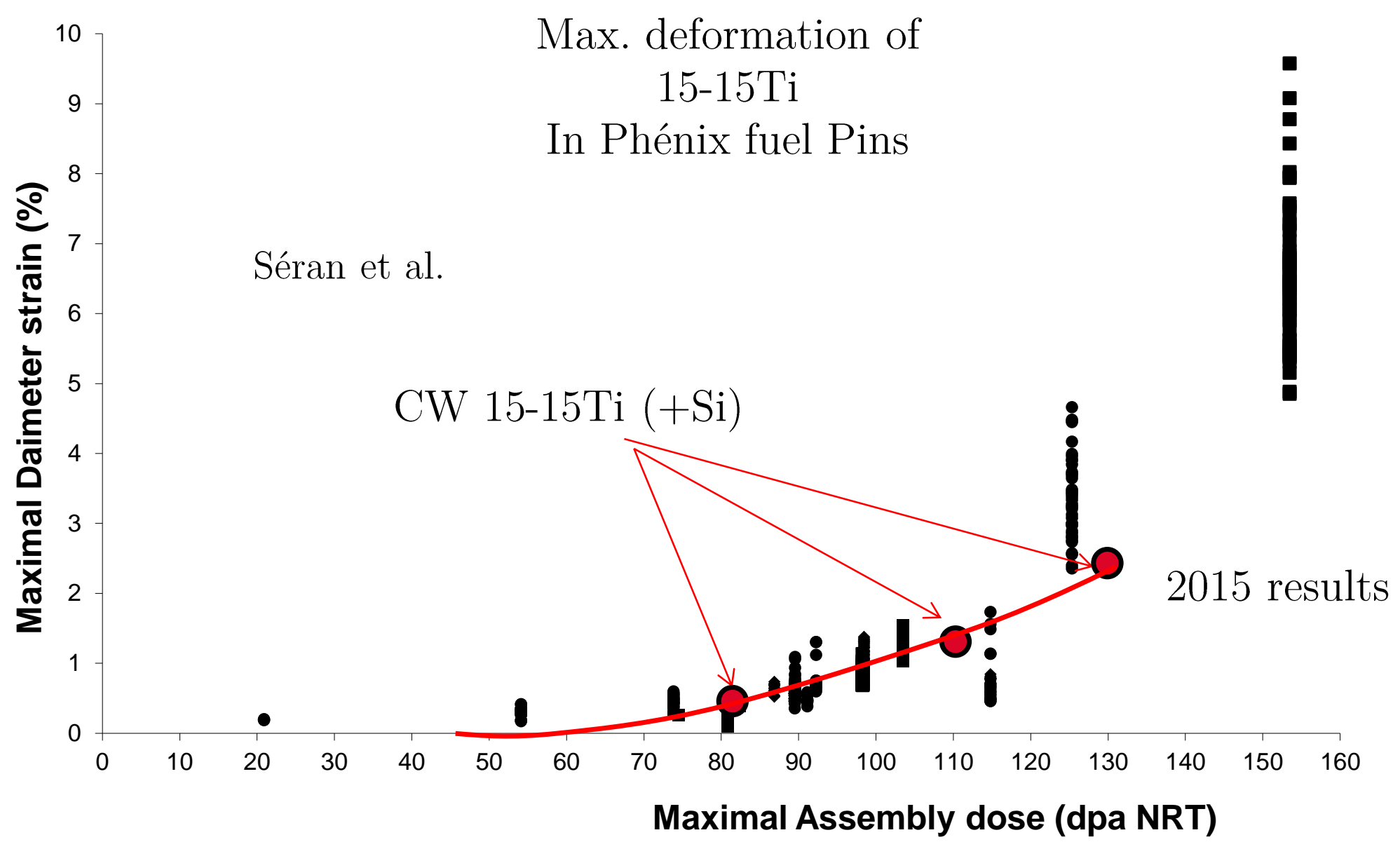




\section{Cea TEM OBSERVATION}

\section{MIN口S}



Standard 15-15Ti (sample) $88 \mathrm{dpa}-467^{\circ} \mathrm{C}$

$\mathrm{G}$ phase $\left(\mathrm{M}_{6} \mathrm{Ni}_{\mathrm{x}} \mathrm{Si}_{\mathrm{y}}\right) / \mathrm{M} 6 \mathrm{C}$ attached to cavities






\section{Cea DISLOCATION LOOPS.}

\section{¿MINDS}

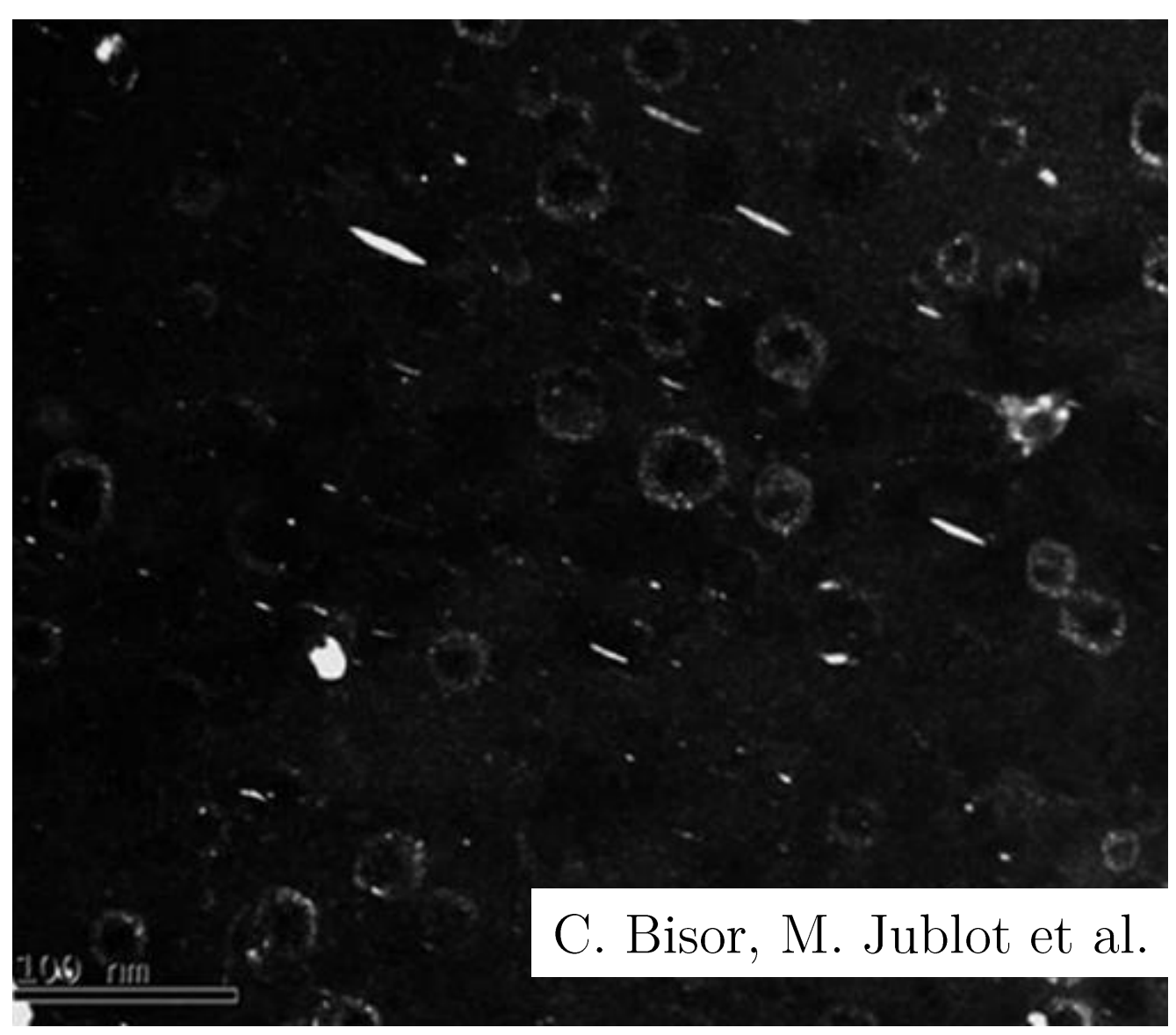




\section{ION IRRADIATION}




\section{Cea JANNUS-SACLAY (EMIR - DMN/SRMP)}

\section{$\because$ MINOS}

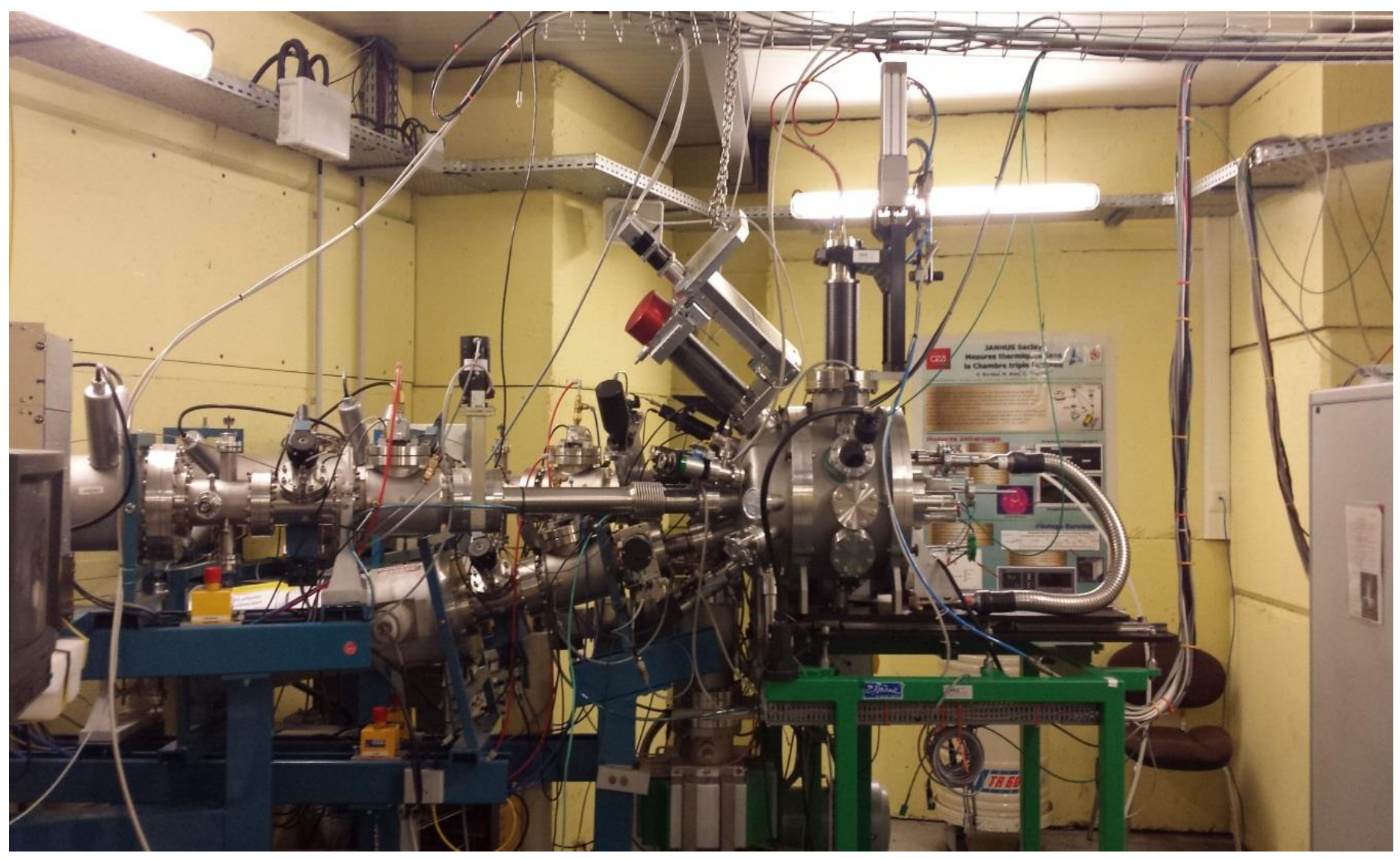




\section{Cea PRINCIPLE}

\section{OMINDS}
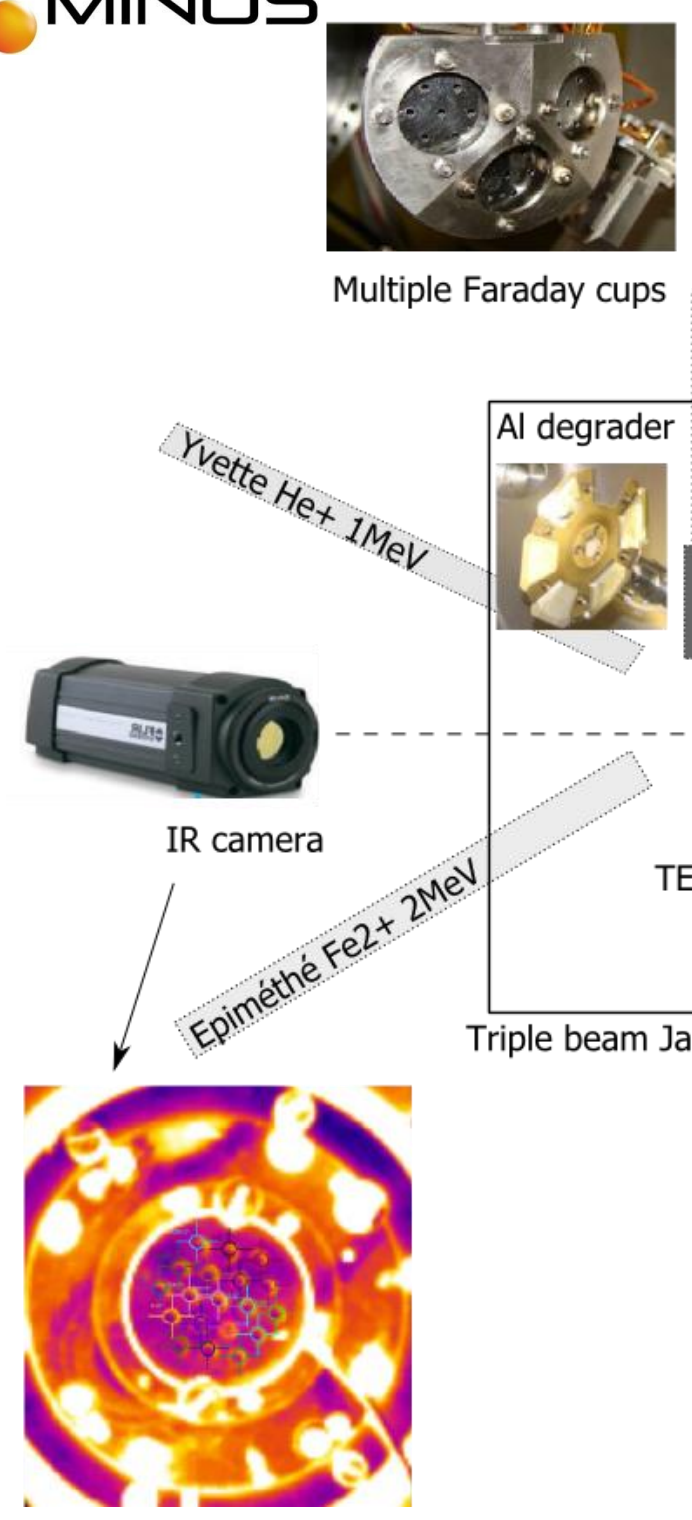



\section{Cea SINGLE BEAM : FE2+ (1MEV) $\mathrm{T}=630^{\circ} \mathrm{C}$}

\section{MINDS}
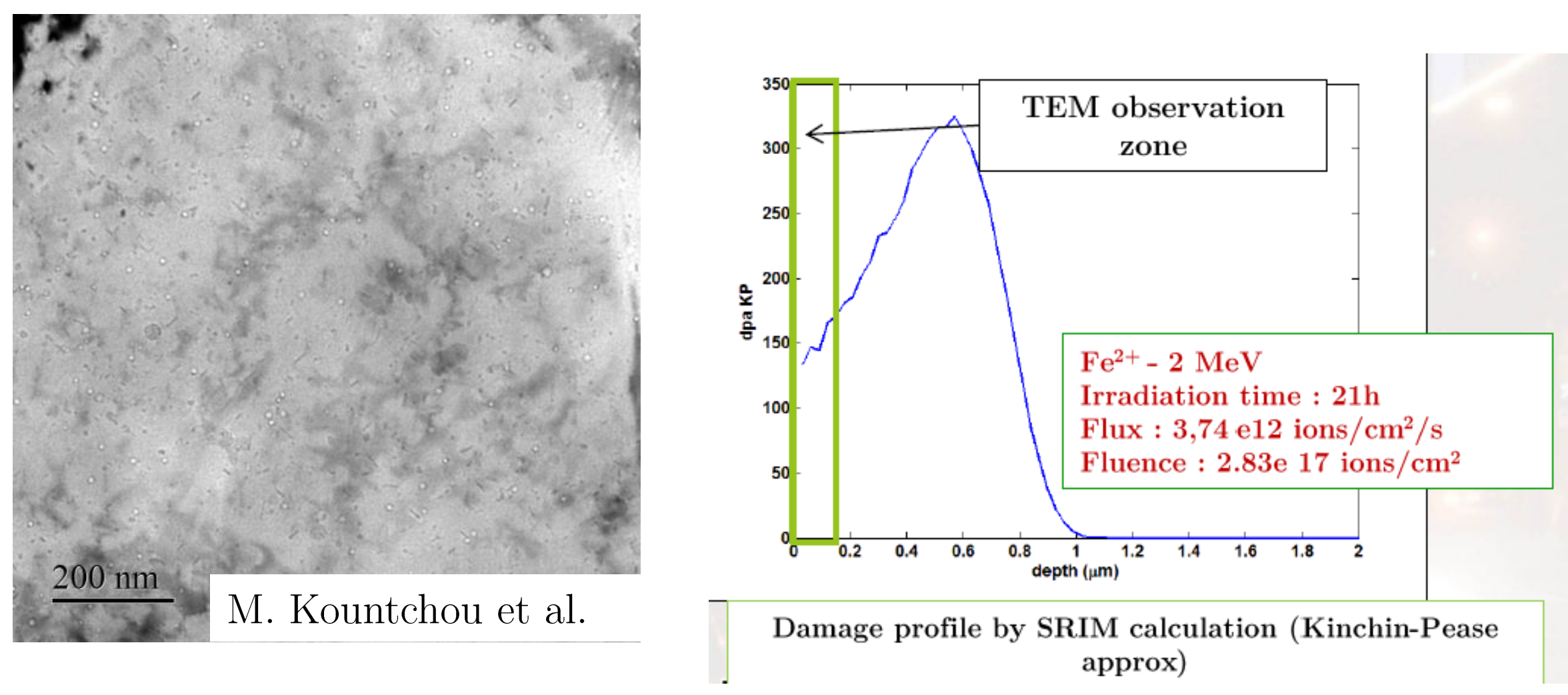


\section{Cea CAVITIES : DUAL BEAM FE ${ }^{2+}-\mathrm{HE}^{2+}\left(\mathrm{T}=630^{\circ} \mathrm{C}\right)$}

\section{MINDS}
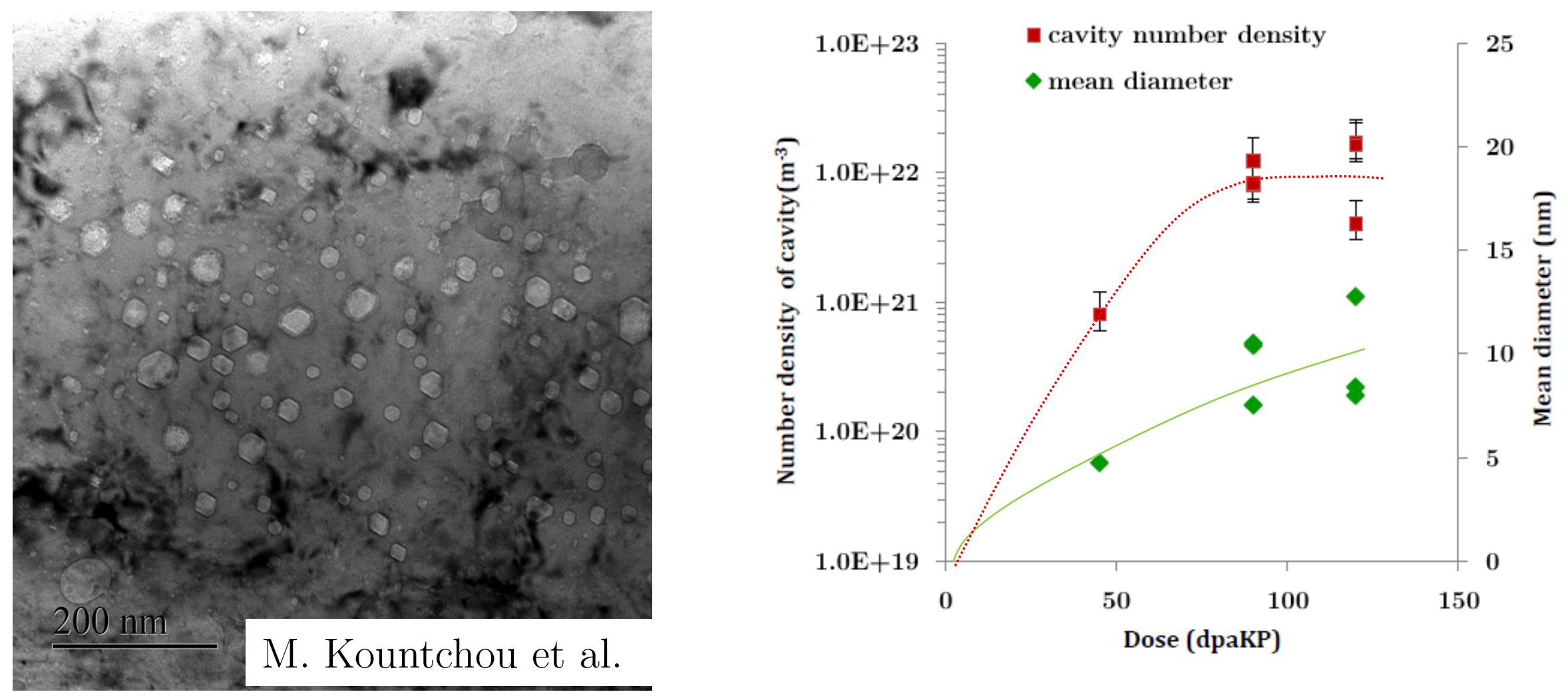


\section{Cea CAVITIES : DUAL BEAM FE2+ - HE2+}

\section{$\because$ MINDS}
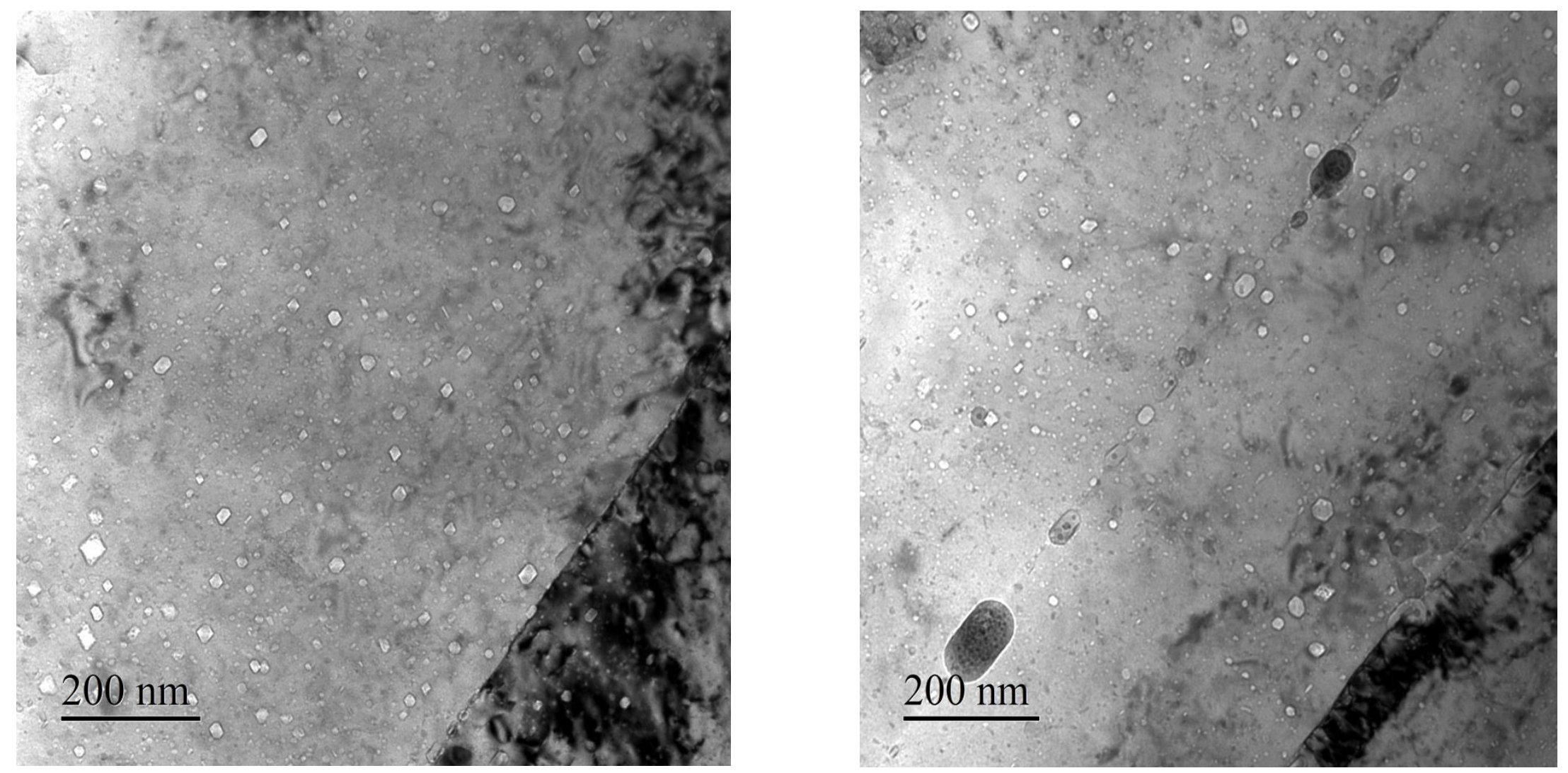


\section{Cea DOSE EFFECT}

\section{¿MINDS}
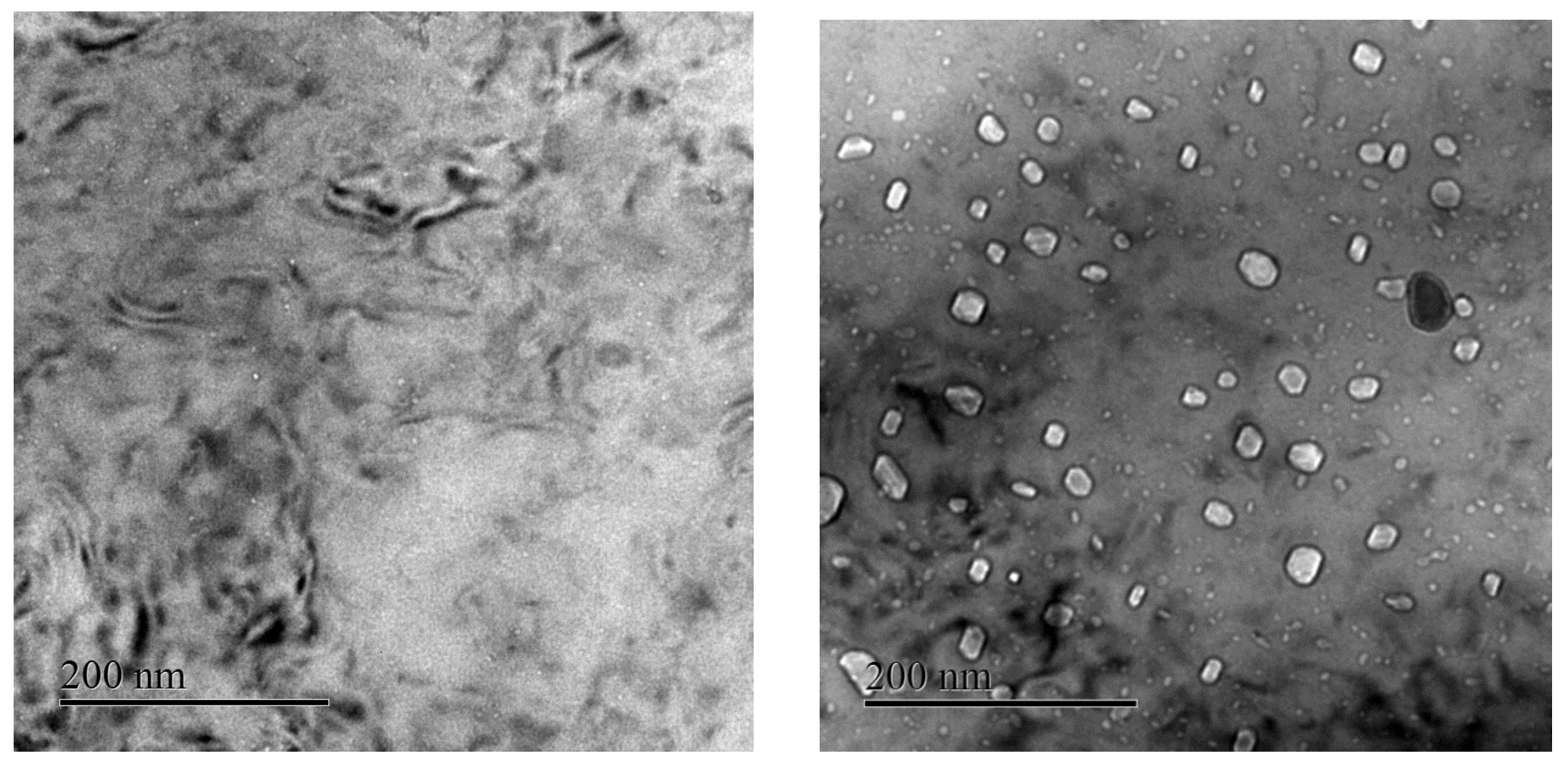

15-15Ti $(\mathrm{Si}+\mathrm{P})\left(\mathrm{Fe}^{2+}+\mathrm{He}^{+}\right), \underline{45 \mathrm{dpaKP}}$

15-15Ti $(\mathrm{Si}+\mathrm{P})\left(\mathrm{Fe}^{2+}+\mathrm{He}^{+}\right), \underline{90 \mathrm{dpaKP}}$ 


\section{Cea DISLOCATION LOOPS}

\section{MIN口S}
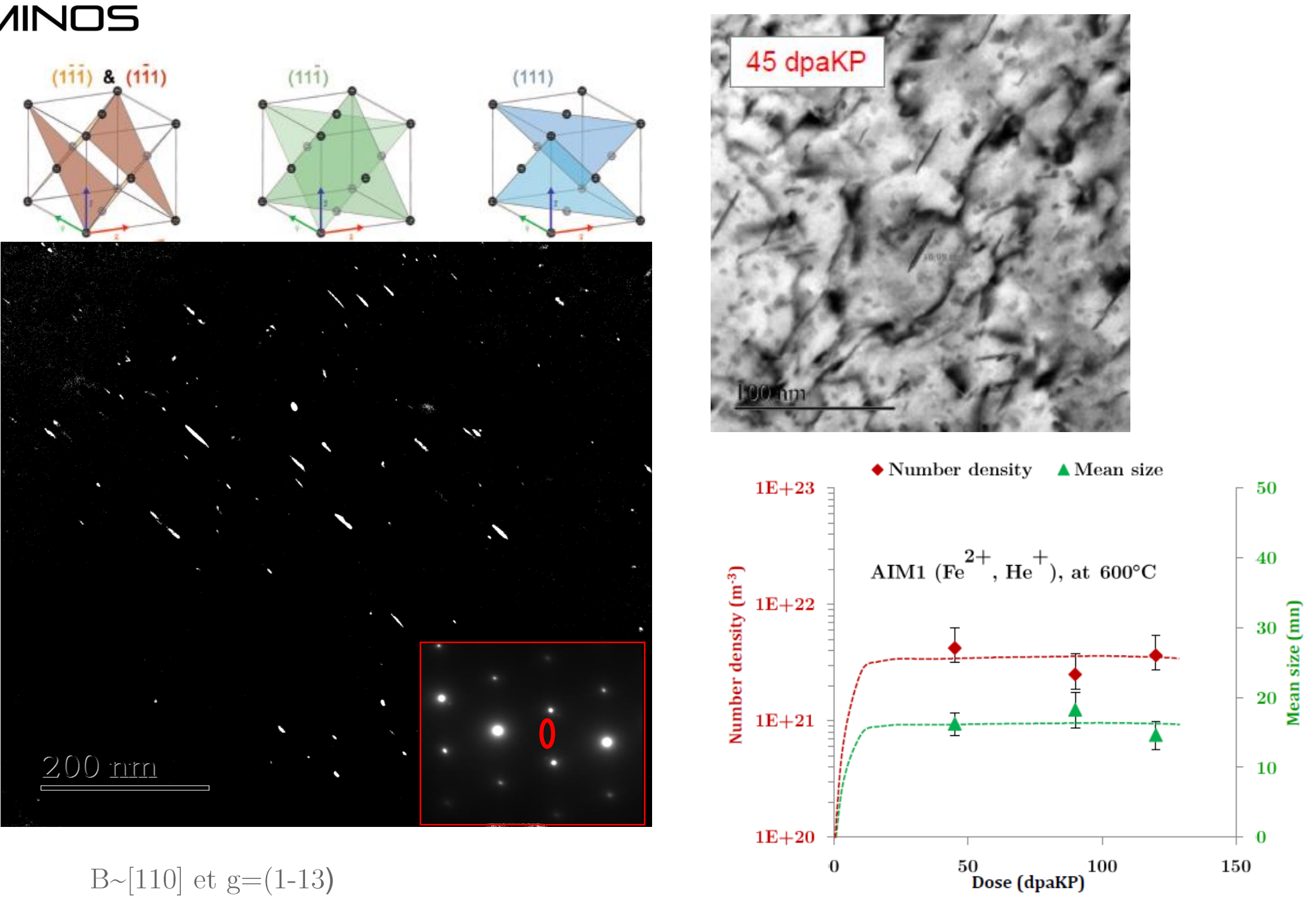

$\mathrm{B} \sim[110]$ et $\mathrm{g}=(1-13)$ 


\section{Cea DISLOCATION}

\section{$\because \mathrm{MINDS}$}

o Still here!

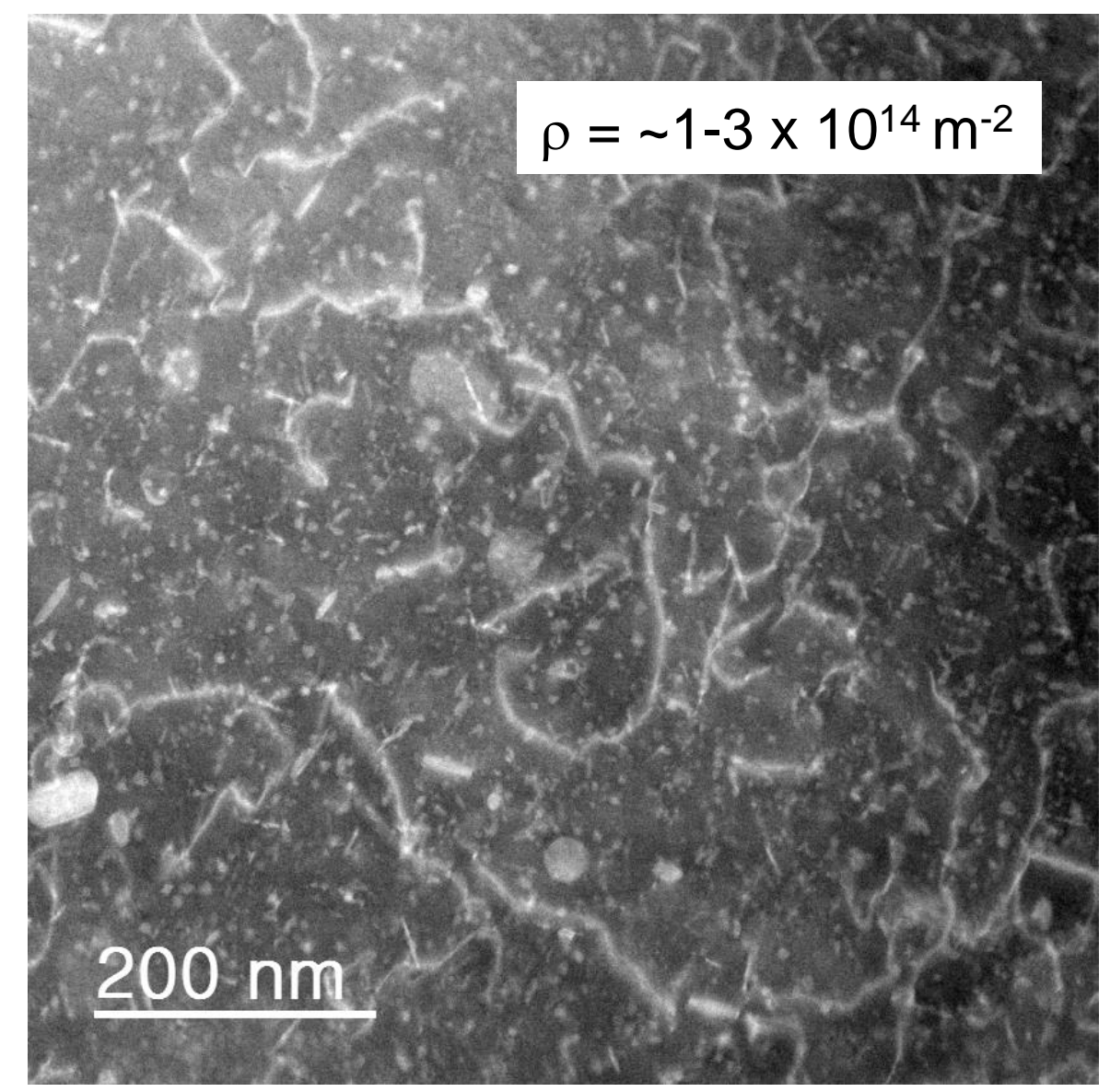

M. Kountchou + A. Etienne (GPM Rouen)

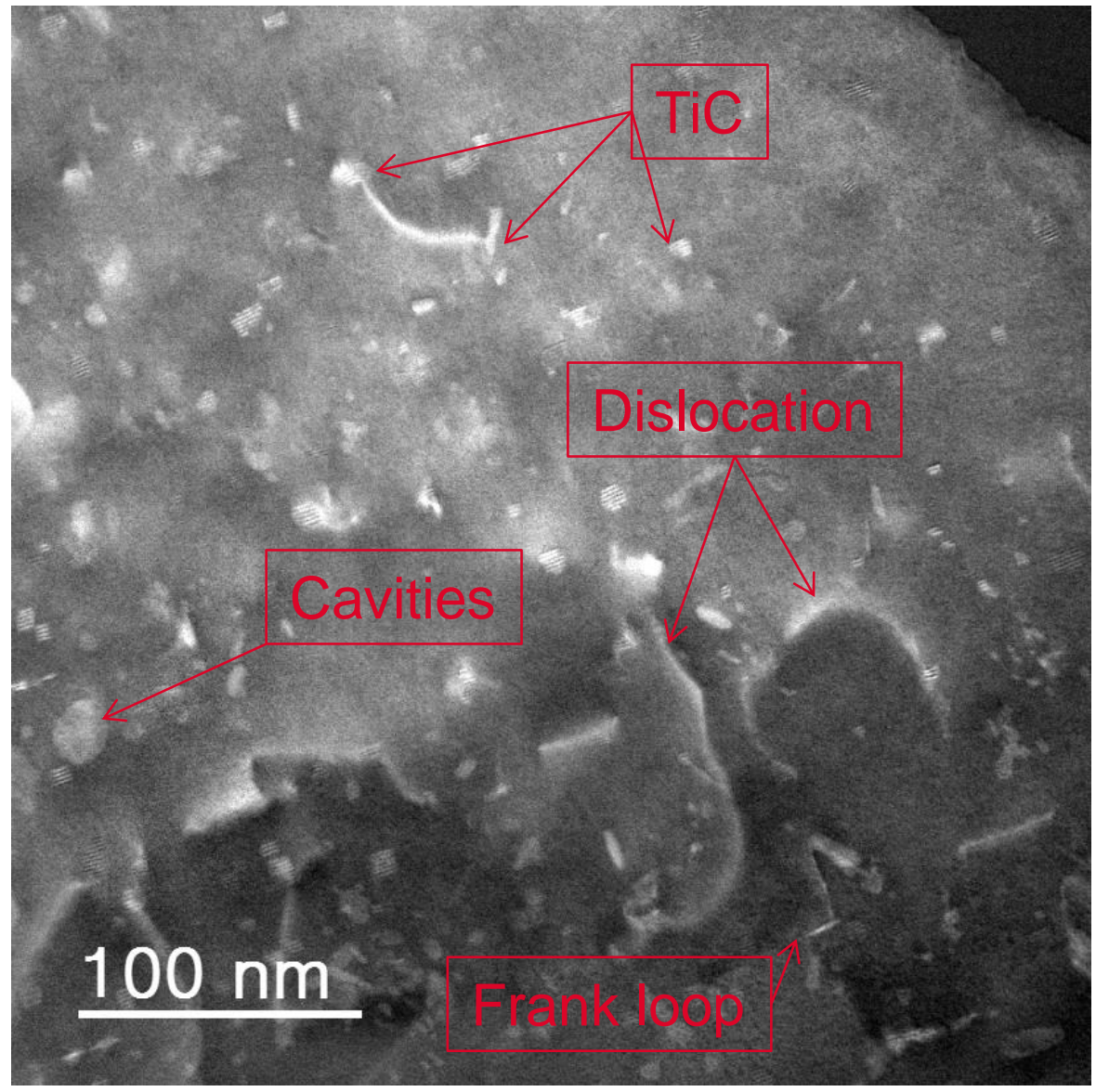




\section{Cea NANO-PRECIPITATION MC}

\section{$\because$ MINOS}

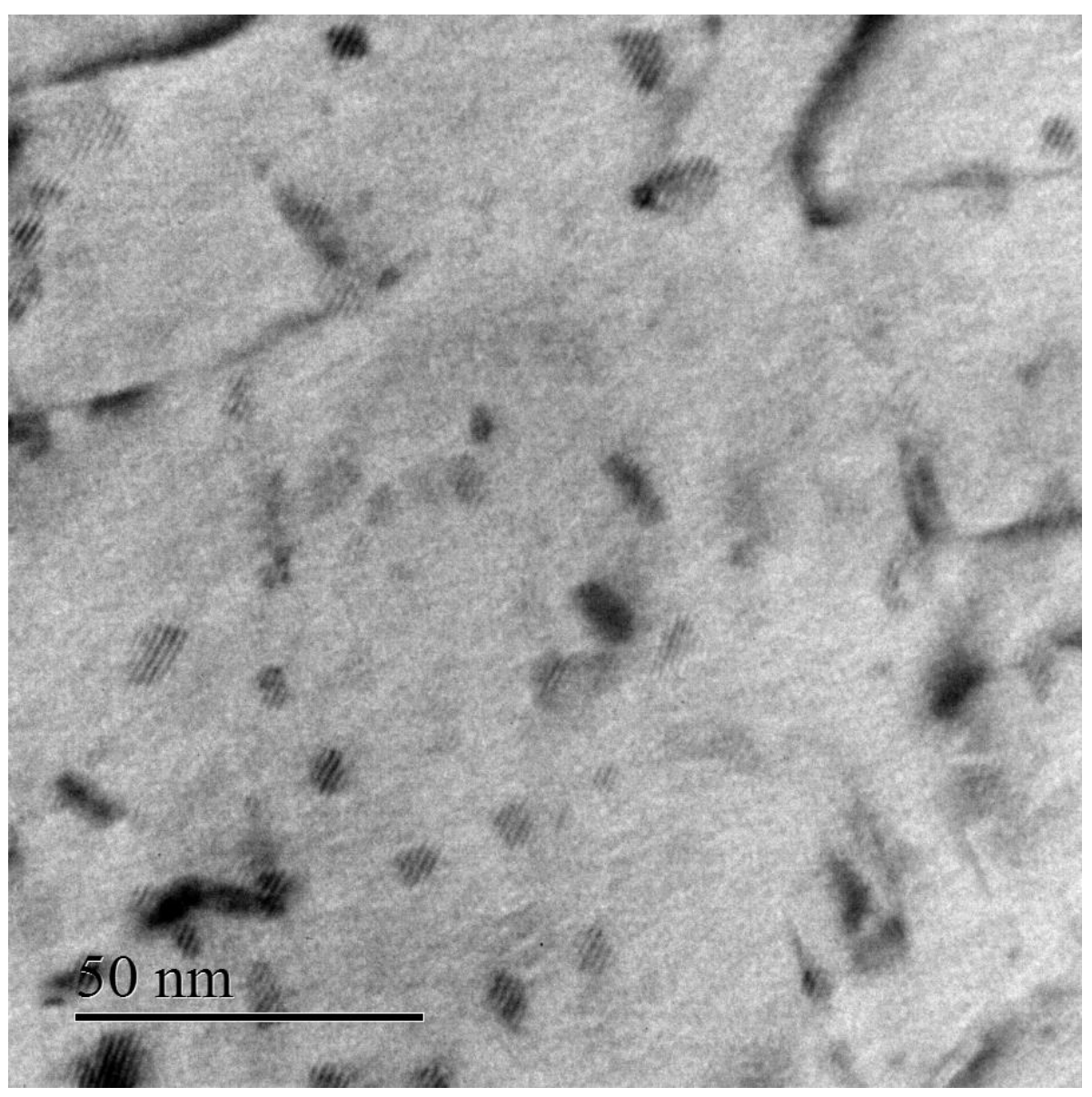




\section{Cea ASSOCIATION CAVITIES-TIC}

\section{$\because$ MINOS}

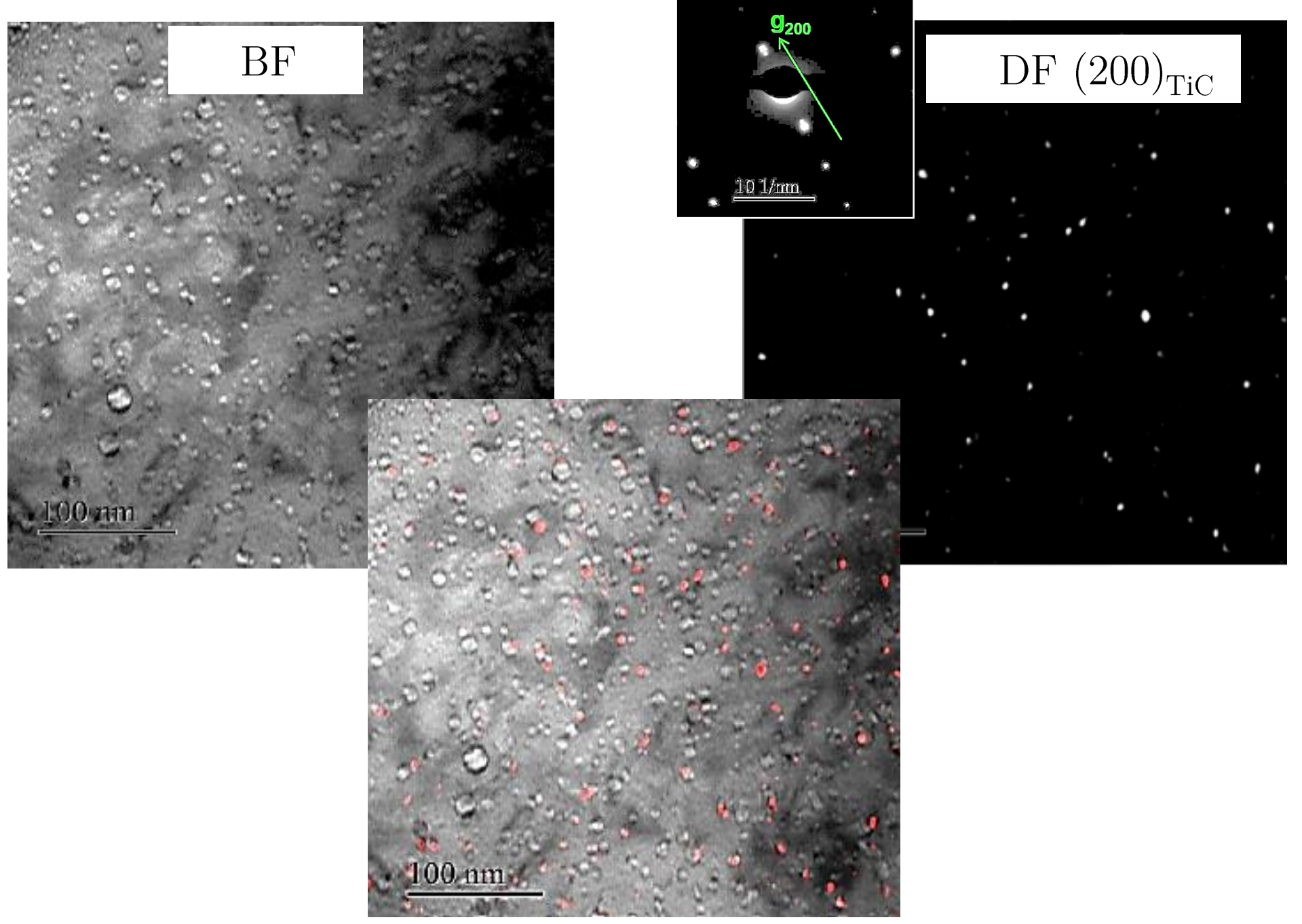




\section{MODELING}




\section{Cea CLUSTER-DYNAMICS MODEL}

\section{MIN口S}

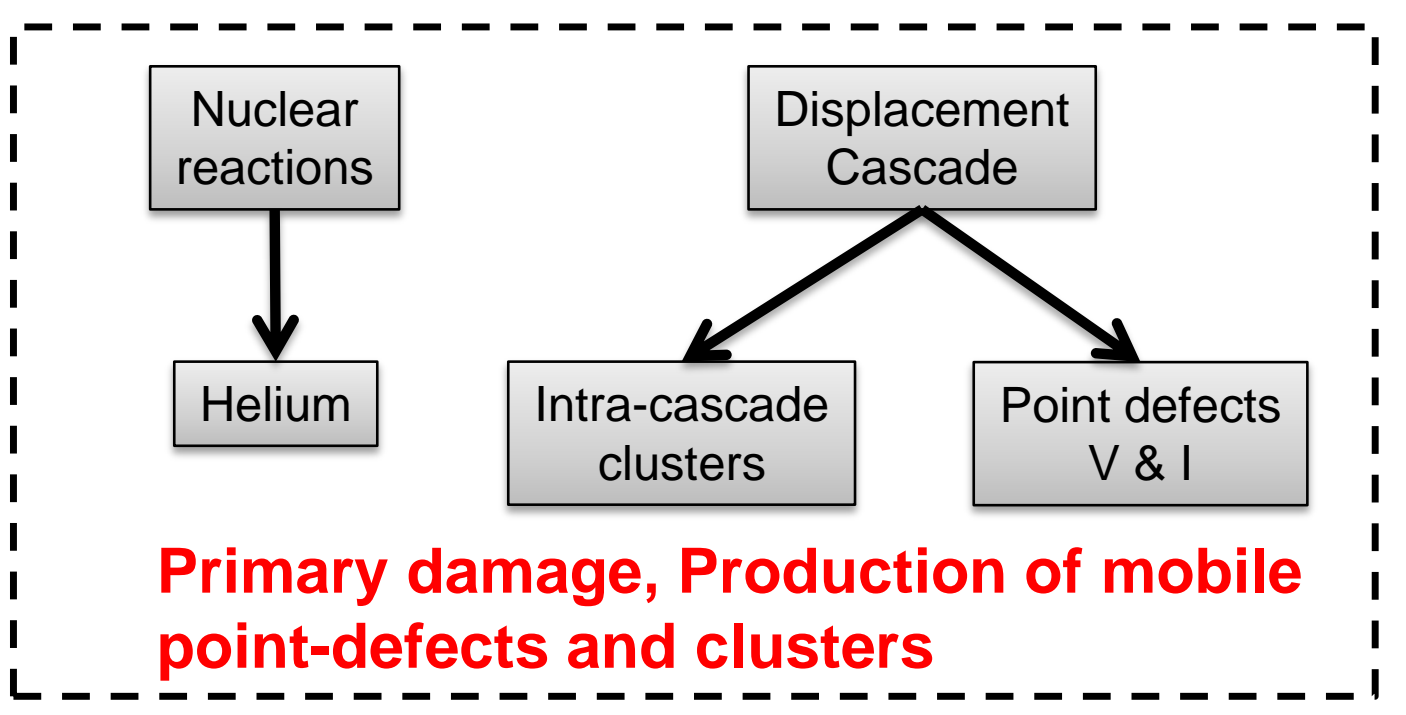

CRESCENDO Framework (T. Jourdan et al.CEA/EDF) 


\section{Cea CLUSTER-DYNAMICS MODEL}

\section{MINDS}


$$
\frac{\mathrm{d} C_{n}}{\mathrm{~d} t}=\sum_{m \in \mathcal{M}} J_{n-m, n}-\sum_{m \in \mathcal{M}} J_{n, n+m}
$$

$\mathrm{m}$ : mobile species

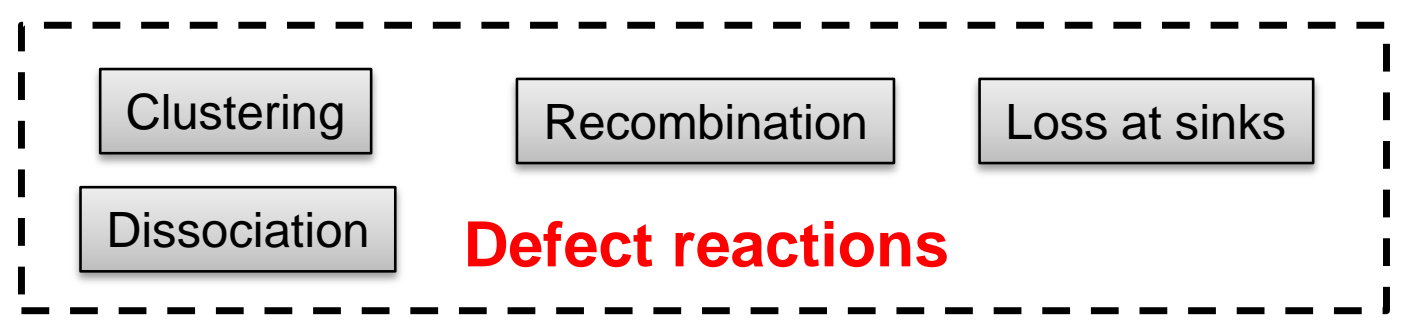




\section{Cea CLUSTER-DYNAMICS MODEL}

\section{MIN口S}

15-15Ti $(+\mathrm{Si}+\mathrm{P})$

Annealed

B Rouxel et al.

Submitted to JNM

IVEM (ANL)

$1 \mathrm{Mev} \mathrm{Kr} 2^{+}$

$600^{\circ} \mathrm{C}$


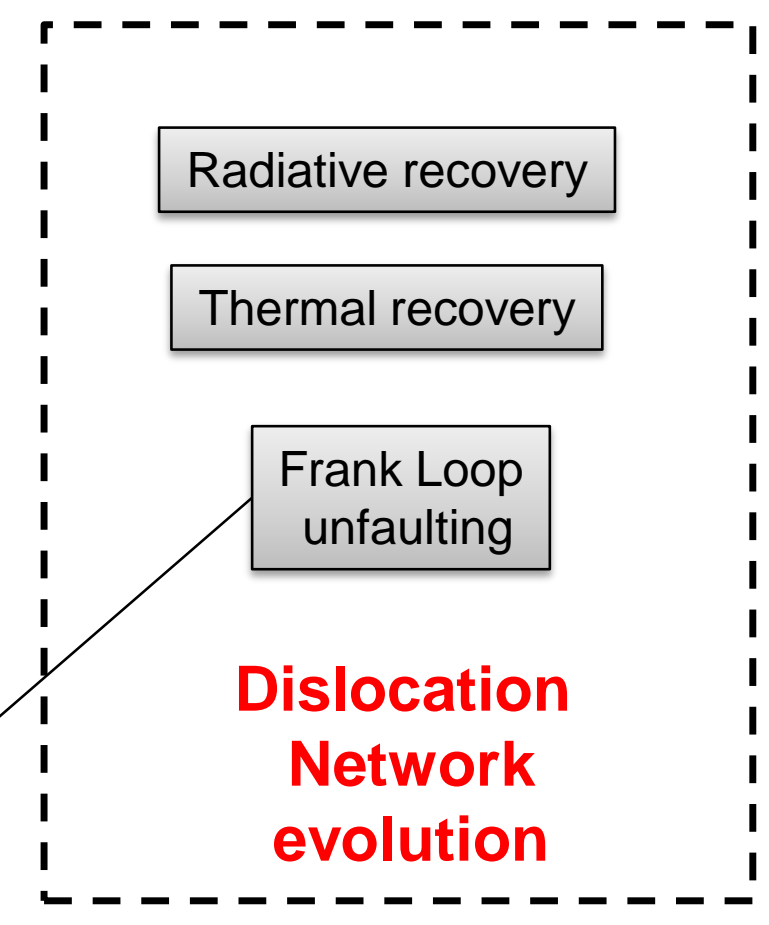




\section{Cea CLUSTER-DYNAMICS MODEL}

\section{MINDS}

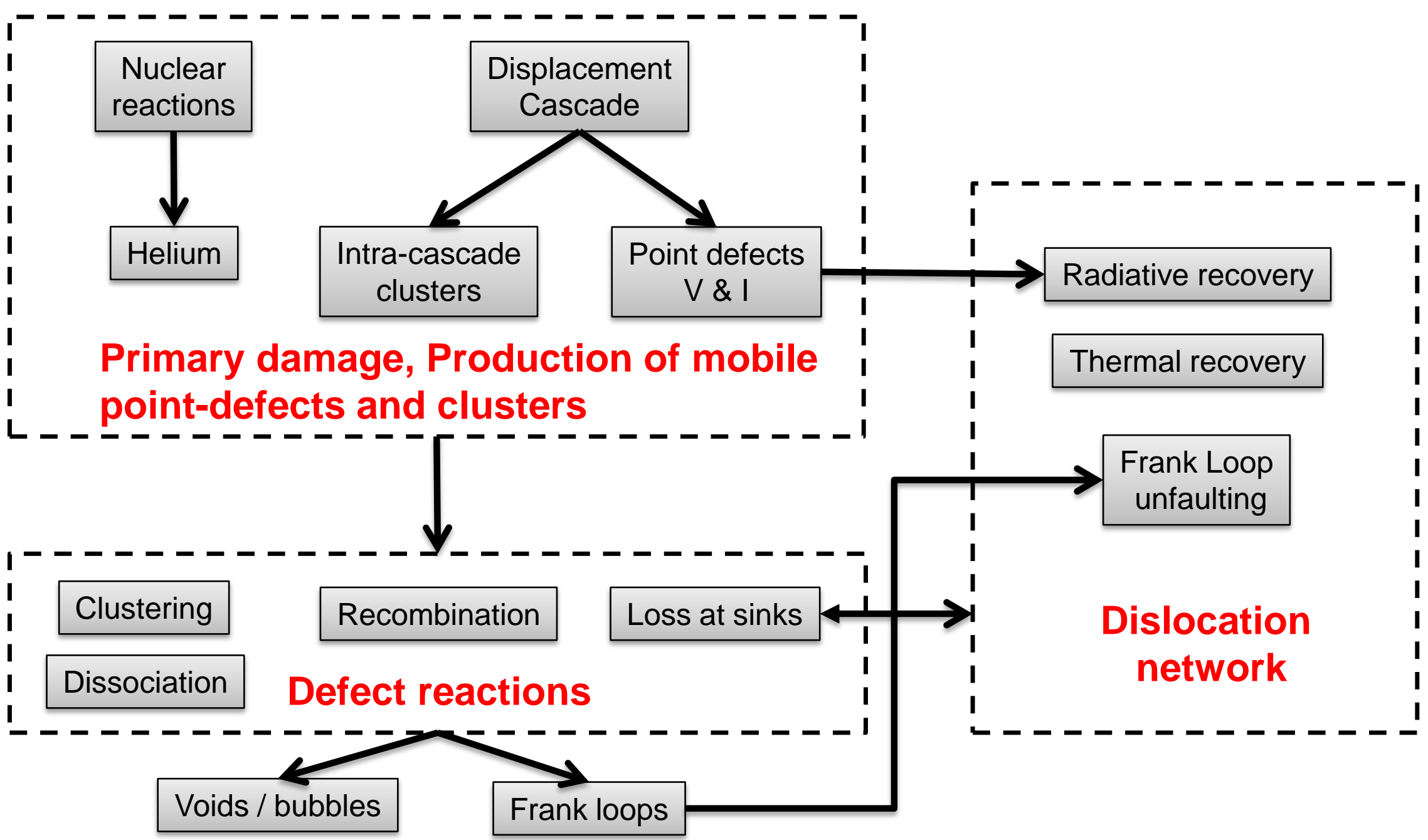




\section{Cea CLUSTER-DYNAMICS MODEL}

\section{MINDS}

\begin{tabular}{|c|c|}
\hline $\begin{array}{l}a \\
V_{\text {at }} .\end{array}$ & $\begin{array}{l}\text { lattice parameter } \\
\text { atomic volume }\end{array}$ \\
\hline$c_{b h, 1}$ & first constant for Bardeen Herring sources \\
\hline$c_{b h, 2}$ & second constant for Bardeen Herring sources \\
\hline$c_{a, 1}$ & first constant for annihilation of dipoles \\
\hline$c_{a, 2}$ & second constant for annihilation of dipoles \\
\hline$\frac{\sigma}{E_{2 i, j}}=$ & $\begin{array}{l}\text { standard deviation of the dislocation distribution } \\
\text { binding energy of di-interstititials }\end{array}$ \\
\hline$E_{2 v, v}^{b}$ & binding energy of di-vacancies \\
\hline$E_{i}^{+-}$ & 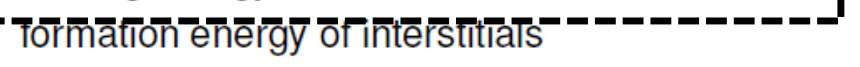 \\
\hline$E_{v}^{f}$ & formation energy of vacancies \\
\hline$\mu$ & shear modulus \\
\hline$v$ & Poisson's ratio \\
\hline$\gamma$ & stacking fault energy \\
\hline  & Burgers ㄱector for Frank Lops \\
\hline$Z_{i}^{\prime}$ & absorption efficiency of Frank loops for \\
\hline$Z_{v}^{\prime}$ & absorption efficiency of Frank loops for vacan \\
\hline$Z_{i}^{d}$ & absorption efficiency of dislocations for interstiti \\
\hline$Z_{v}^{d}$ & absorption efficiency of dislocations for vacancies \\
\hline$E_{i}^{m}$ & migration energy of interstitials \\
\hline$E_{v}^{m}$ & migration energy of vacancies \\
\hline$D_{0, i}$ & diffusion prefactor for interstitials \\
\hline$D_{0, v}$ & diffusion prefactor for vacancies \\
\hline$-\nabla \nabla_{i}^{-}$ & - retaxánoñ vorume orinterstitais \\
\hline$\Delta V_{v}$ & relaxation volume of vacancies \\
\hline$\rho$ & initial dislocation density \\
\hline
\end{tabular}

Dislocation evolution

Binding energy for

emission rate

Bias, absorption efficiency
Diffusion coefficients of
defects 


\section{CEA ELECTRON IRRADIATION}

Exp Data from Dubuisson et al.

- Standard 15-15Ti

- Annealed $1150^{\circ} \mathrm{C}-10 \mathrm{mn}$

- 475 and $500^{\circ} \mathrm{C}$

- $\quad \sim 3.510^{-3} \mathrm{dpa} / \mathrm{s}$

- Beam direction <110>

- Foil thickness ( 300nm)



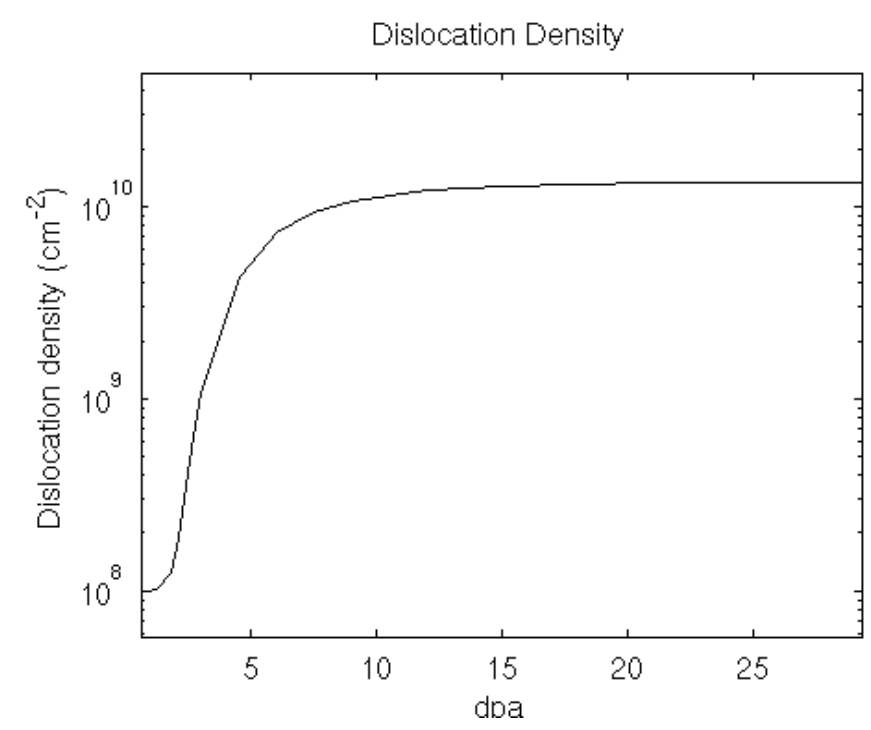

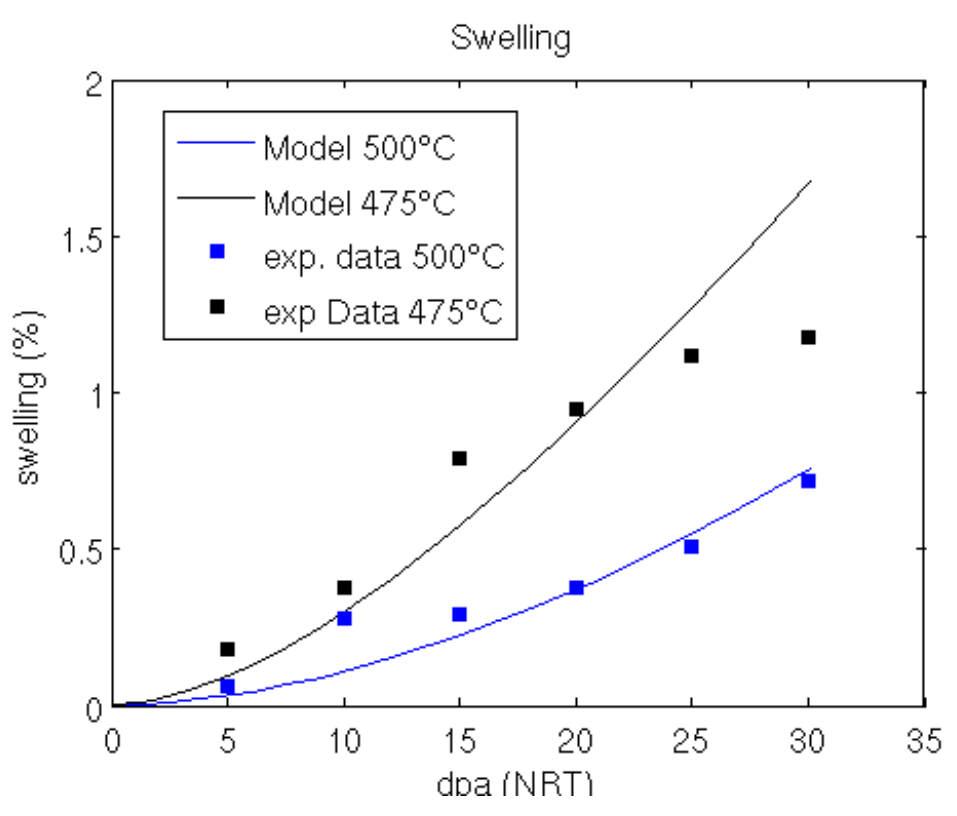




\section{Cea CLUSTER DISTRIBUTION}

\section{MINDS}






\section{CEA FLUX - DPA RATE EFFECT}

\section{MINDS}

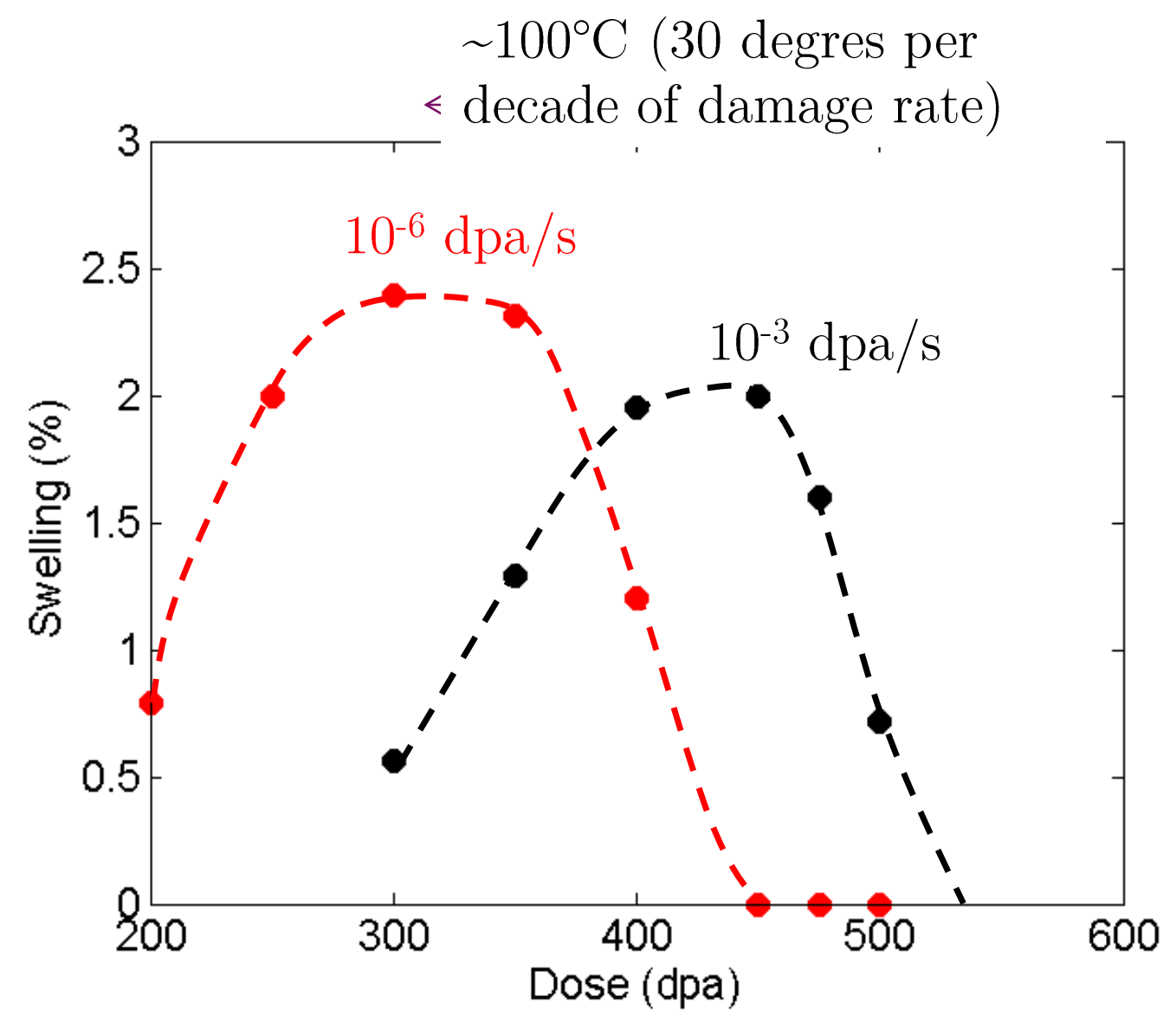

Temperature Shift 


\section{Cea EFFECT OF COLD WORKING}

\section{MINDS}


Incubation dose ! 


\section{IMPACT ON MECHANICAL PROPERTIES}




\section{Cea IMPACT OF IRRADIATION ON MECHANICAL PROPERTIES}

\section{MINDS}

○ Axial tensile tests (in hot cells)

Softening at high

CW 15-15Ti (+Si)

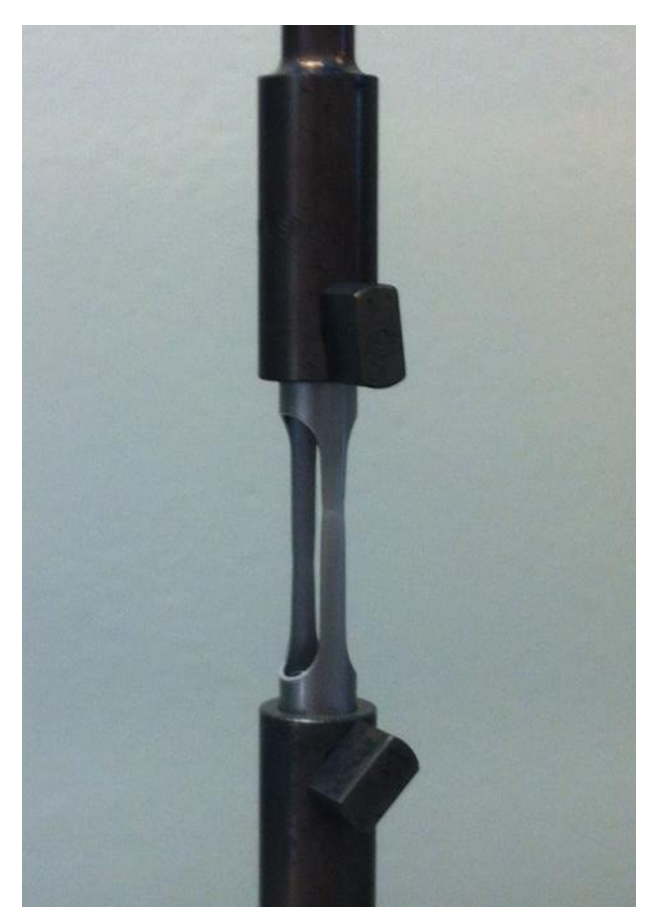

Hardening below $500^{\circ} \mathrm{C}$

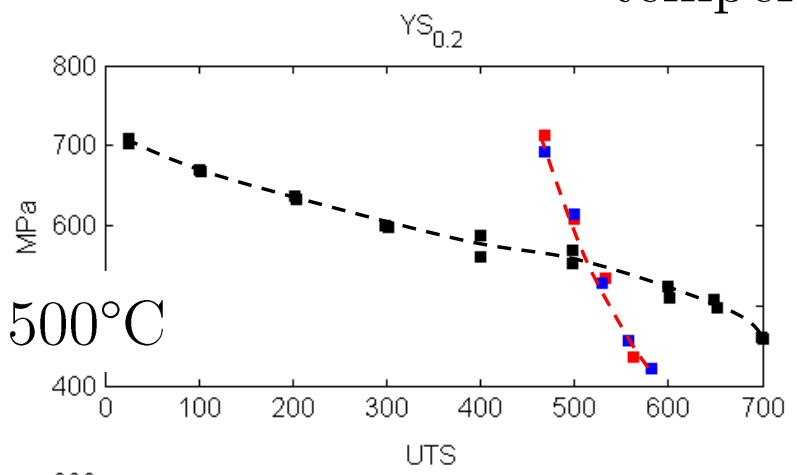

temperature
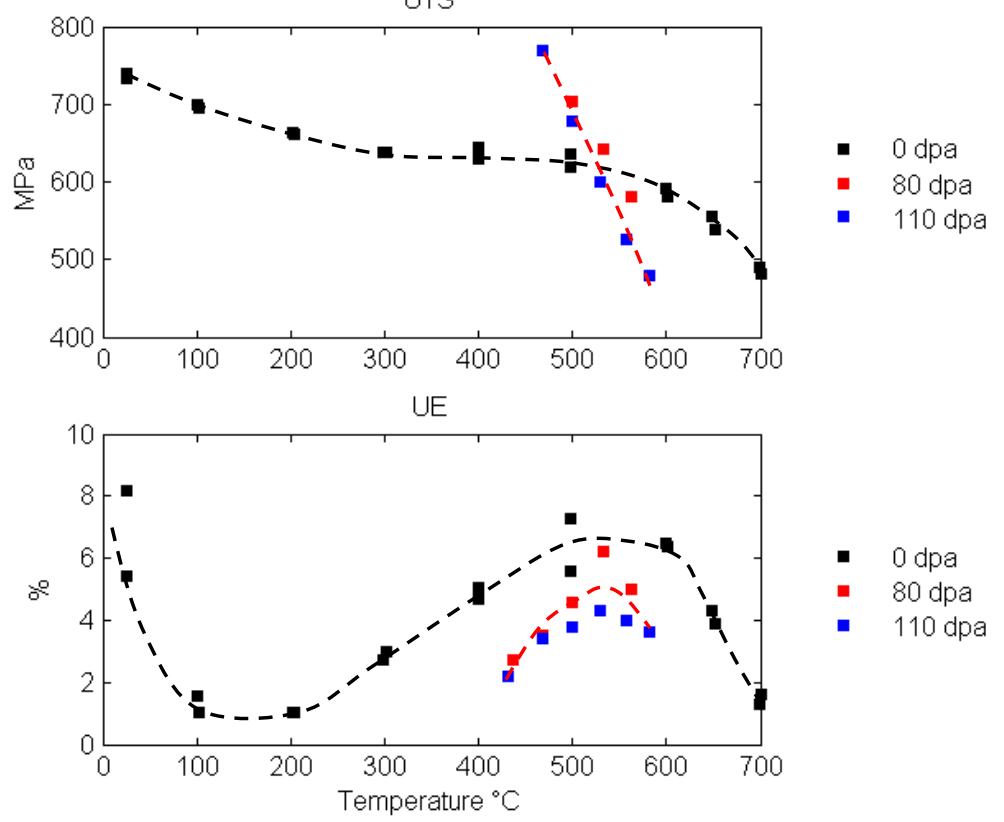

Reduced ductility 


\section{IMPACT OF IRRADIATION ON MECHANICAL PROPERTIES}

\section{MINDS}

- Axial tensile tests in hot cells

○ CW 15-15Ti (low \& high Si, low P)



\section{CONCLUSION}




\section{CONCLUSIVE REMARKS}

\section{MINDS}

o Neutrons are the only way to qualify a real (complex) material in irradiation

o Ions/Electrons are the shortest/cheapest way to perform R\&D (mechanisms understanding, material comparison)

- Model are essential to rationalize experimental data (dose, temperature, dose rate, helium effect)

- But the road towards a mechanistic (multiscale) and predictive model of real alloy like $15-15 \mathrm{Ti}$ is still long 


\section{MINDS}

○ M. Kountchou, B. Rouxel, L Mateus-Freire (Phd CEASaclay)

- JL Seran, M. LeFlem, P. Gavoille, C. Bisor, M. Jublot, E. Piozin, P. Bottin (CEA-Saclay DEN/DANS/DMN)

O O. Descombin, T. Martella, L. Loubet (CEACadarache DEN/DANS/DEC-LECA)

○ M. Masson (CEA-Marcoule, Phénix)

○ E. Buravand (CEA-Marcoule, Atalante)

○ Jannus Team : (CEA-Saclay SRMP \& SRMA) 